\title{
All the trinomial roots, their powers and logarithms from the Lambert series, Bell polynomials and Fox-Wright function: illustration for genome multiplicity in survival of irradiated cells
}

\section{Dževad Belkić ${ }^{1,2}$}

Received: 29 August 2018 / Accepted: 15 November 2018 / Published online: 5 December 2018 (c) The Author(s) 2018

\begin{abstract}
All the roots of the general $n$th degree trinomial admit certain convenient representations in terms of the Lambert and Euler series for the asymmetric and symmetric cases of the trinomial equation, respectively. Previously, various methods have been used to provide the proofs for the general terms of these two series. Taking $n$ to be any real or complex number, we presently give an alternative proof using the Bell (or exponential) polynomials. The ensuing series is summed up yielding a single, compact, explicit, analytical formula for all the trinomial roots as the confluent Fox-Wright function ${ }_{1} \Psi_{1}$. Moreover, we also derive a slightly different, single formula of the trinomial root raised to any power (real or complex number) as another ${ }_{1} \Psi_{1}$ function. Further, in this study, the logarithm of the trinomial root is likewise expressed through a single, concise series with the binomial expansion coefficients or the Pochhammer symbols. These findings are anticipated to be of considerable help in various applications of trinomial roots. Namely, several properties of the ${ }_{1} \Psi_{1}$ function can advantageously be employed for its implementations in practice. For example, the simple expressions for the asymptotic limits of the ${ }_{1} \Psi_{1}$ function at both small and large values of the independent variable can be used to readily predict, by analytical means, the critical behaviors of the studied system in the two extreme conditions. Such limiting situations can be e.g. at the beginning of the time evolution of a system, and in the distant future, if the independent variable is time, or at low and high doses when the independent variable is radiation dose, etc. The present analytical solutions for the trinomial roots are numerically illustrated in the genome multiplicity corrections for survival of synchronous cell populations after irradiation.
\end{abstract}

Dževad Belkić

Dzevad.Belkic@ki.se

1 Department of Oncology-Pathology, Karolinska Institute, P.O. Box 260, 17176 Stockholm, Sweden

2 Department of Medical Radiation Physics and Nuclear Medicine, Karolinska University Hospital, P.O. Box 260, 17176 Stockholm, Sweden 
Keywords Trinomial roots · Trinomial equations $\cdot$ Lambert functions $\cdot$ Euler series

\section{Contents}

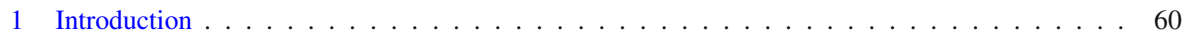

1.1 Trinomial roots . . . . . . . . . . . . . . . . . . . . . 60

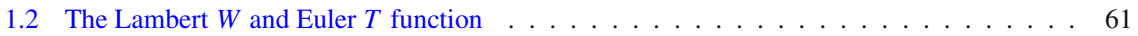

1.3 Applications using trinomial roots . . . . . . . . . . . . . . . . . . . 65

2 The complete Bell polynomials . . . . . . . . . . . . . . . . . . . . . . . . 65

3 The cyclic indicator polynomials . . . . . . . . . . . . . . . . . . . 68

4 The partial Bell polynomials . . . . . . . . . . . . . . . . . . . . . . . . . 69

5 Derivatives of any analytical function raised to an arbitrary power . . . . . . . . . . . 71

6 An arbitrary power of a MacLaurin series of any function . . . . . . . . . . . . . . . . . 72

7 The Lambert series solution for all the roots of trinomial equations . . . . . . . . . . . . . . . 74

8 All the trinomial roots in terms of the Bell polynomials . . . . . . . . . . . . . . . . . . . . 77

9 From multi- to uni-variate polynomials for trinomial roots . . . . . . . . . . . . . . . 78

10 All the trinomial roots by a series in terms of the Pochhammer symbols . . . . . . . . . . 80

11 Arbitrary real- or complex-valued powers of trinomial roots . . . . . . . . . . . . . . 81

12 Logarithmic function of trinomial roots . . . . . . . . . . . . . . . . . . . 83

13 Trinomial roots in terms of the confluent Fox-Wright function . . . . . . . . . . . . . . . . 84

14 Convergence radius of the series for trinomial roots . . . . . . . . . . . . . . . . . 85

15 An illustration in radiobiology for radiotherapy . . . . . . . . . . . . . . . . . . . . 88

16 Discussion and conclusions . . . . . . . . . . . . . . . . . . . . . . . 91

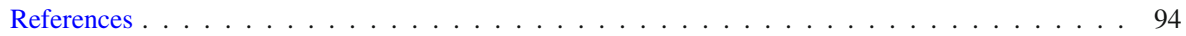

\section{Introduction}

Since the topics of the trinomial roots and the Lambert function have historically been tightly intertwined, we shall subdivide this introductory section into two parts, one dealing with the former and the other with the latter subject.

\subsection{Trinomial roots}

The theme of the roots of trinomials has a remarkable history beginning with Lambert in 1758 [1,2], followed by Euler in 1777 [3,4] and continued by many authors during the past 260 years to the present. In particular, it is from finding all the trinomial roots that the important subject of the Lambert $W$ and Euler $T$ functions emerged in the literature. Research on trinomial roots resulted in numerous reports, some of which are given in Refs. [5-52] (1851-2018). Presently, we primarily focus upon derivations of the analytical formulae for all the roots of trinomials through the series developments using the Bell polynomials [53] (1934) and the Fox-Wright function [54-58] (19331961). The Bell (or exponential) polynomials arise in obtaining the closed expressions for general derivatives of functions. For example, the Faà di Bruno formula [5961] (1855-2002) for the $n$th derivative of composite functions can be derived by using the Bell polynomials, as shown by Riordan [62,63] (1946,1978). The FoxWright function ${ }_{n} \Psi_{m}$ is an extension of the generalized Gauss hypergeometric function ${ }_{n} F_{m}$. The confluent Fox-Wright function ${ }_{1} \Psi_{1}$ is the generalized Kummer confluent hypergeometric function ${ }_{1} F_{1}$. While the series for ${ }_{1} F_{1}$ in powers of its independent 
variable ( say $x$ ) is known to converge at any finite $x(|x|<\infty)$, the corresponding series for ${ }_{1} \Psi_{1}$ in $x$ converges only within its convergence radius $R(|x|<R)$.

In 1777, Euler [3] found a series for all the roots of the symmetrized form of the trinomial characteristic equation. Subsequently, over a long period of time, using various methods, the Euler formula has been proven by a number of authors ranging from McClintock in 1895 [11] to Wang in 2016 [50]. We presently give yet another proof of the Euler formula for all the trinomial roots by deriving the explicit expression for the general expansion coefficient in terms of the complete Bell polynomials $B_{n}$. Moreover, transforming these multivariate to univariate polynomials, the expansion coefficients are reduced to the binomial coefficients and the Pochhammer symbols $(a)_{n}$. Finally, the obtained series is explicitly summed up with the result given by the confluent Fox-Wright ${ }_{1} \Psi_{1}$ function.

The Fox-Wright functions ${ }_{n} \Psi_{m}$ [54-58] and its generalizations have been used in a number of studies on different subjects and a few articles are listed in Refs. [45-47,6467] (1994-2007). The usefulness of the analytical formula for trinomial roots in terms of the confluent Fox-Wright function ${ }_{1} \Psi_{1}$ is in the possibility to exploit the known asymptotic behaviors of the ${ }_{1} \Psi_{1}$ function at both small and large values of its independent variable $x$. This is exemplified in the present illustration of the trinomial roots encountered in a radiobiological model for cell survival after exposure to radiation.

\subsection{The Lambert $W$ and Euler $T$ function}

The Lambert and Euler functions, with their most frequently encountered properties, have thoroughly been reviewed in the literature. Therefore, all that is given in this subsection is mainly a complement to the existing compilations of the bibliography on this subject matter. Despite numerous entries in the cited publications, the present list of references is still far from being exhaustive due to a huge number of reported studies. Because of the versatile nature of applications of these two functions in various disciplines, we will categorize the selected articles according to their research branches.

The Lambert $W$ function [1,2] and the related Euler $T$ function $[3,4]$ play a very important role across interdisciplinary research. These two functions are the multi-valued solutions of the transcendental equations $y=x \mathrm{e}^{x}[\therefore x=W(y)]$ and functions $y=x \mathrm{e}^{-x}[\therefore x=T(y)]$. They arise from a linear-exponential, or equivalently, linear-logarithmic equations for the unknown, sought quantity. This special combination of the two elementary functions describes two different behavioral patterns (linear and exponential or linear and logarithmic) that a large number of phenomena share in vastly different fields. The underlying common physical, chemical or biological effects behind a linkage of a linear with an exponential term is often related to two different stages of a complete process of time-evolution of a generic dynamical system. These stages might compete with each other, or they could correspond to a slow and a fast component of the whole developmental process, or they could be associated with the two complementary mechanisms, etc. Such two components may characterize e.g. the rise and fall of the studied observables (experimentally measurable quantities) that describe the behavior of a system in varying environmental conditions under the influence of an external agent. For example, a system of coupled differential rate equations from chemical kinetics (that cannot be solved exactly by analytical 
means) can be approximately reduced (within a quasi-stationary state assumption) to a linear-exponential or linear-logarithmic transcendental equation whose exact solution is the Lambert function. This occurs in the Michaelis-Menten formalism [68] (1913) for enzyme catalysis in the Briggs-Haldane setting [69] (1925). The same linear-exponential pattern behavior is routinely encountered in many systems whose time evolution obeys differential or difference equations. Such time evolution is often accompanied with time delays, in which case the delayed differential equations are used, and these end up with a linear-exponential transcendental equation which yields exactly the Lambert function.

Of course, these transcendental equations can be solved by numerical means (e.g. by the Newton iteration). However, the possibility of obtaining the exact analytical solution of such equations, e.g. through the Lambert function, is appealing. The reason is that a closed, analytical form of a function is invaluable as it provides the necessary asymptotic forms both at small and large values of the independent variable. Such asymptotes govern the development of the system in the two extreme conditions and provide a way to control and, indeed, predict the behavioral patterns. In the last 20 years, the inter-disciplinary literature witnessed an ever increasing interest in the Lambert function. It is anticipated that this enviable trend will be pursued in the next 20 years and beyond.

The mentioned circumstances embodied through the linear-exponential mathematical form in the transcendental equations are ubiquitous and this is the main reason for the universal applicability of the Lambert function in distant and seemingly unrelated fields. It would be virtually impossible to enumerate various mechanisms in versatile research branches that could produce the Lambert function as the end result. The number of articles dealing with this remarkable function is enormous, and no review can be exhaustive enough in citing and/or commenting on a greater part the related publications. The present work is no exception, and we shall content ourselves to mention only a smaller fraction of the past contributions to this topic. What makes an investigative result important is its usefulness to a wider circle of other researchers over an extended period of time. The Lambert function passed this test of time as testified by an unprecedented use of this function in mathematics, physics, astrophysics, chemistry, biology, medicine, population genetics, ecology, sociology, education, energetics, technology, etc. To help the general reader (with a hope of motivating a further extension of the applications of the Lambert function) and especially due to an unprecedentedly abundant literature, it is deemed instructive to group the publications into several categories.

The first quoted are the originators, Lambert and Euler, with two cited articles per author. Subsequently, general information is collected by quoting books, tabular publications, PhD Theses, reviews, international workshops, websites and posters. This is followed by quoting computational contributions (algorithms, programs, libraries, open source codes) and articles with ceveral quite accurate approximate formulae for the Lambert function.

The next quoted are the publications on the applications of the Lambert and Euler functions in various disciplines, such as mathematics, physics, astrophysics/astronomy, chemistry, biomedicine, ecology, sociology, technology and education. Some of these publications deal exclusively with the Lambert and Euler 
functions, whereas the other studies address a number of features of these functions among the other treated topics.

According to the outlined scheme, the list of publications referring to the transcendental Lambert and Euler functions reads as:

- Lambert's articles On the series solution for trinomial roots [1,2](1758, 1770).

- Euler's articles On the Lambert series for trinomial roots [3,4] (1777, 1783).

- Books Series solutions of algebraic equations, theory of transcendental functions, enumerative combinatorics, population of species, time-delayed systems, etc. [7083] (1906-2016). In particular, Pólya and Szegő [71] (1925) examined the function $y=x \mathrm{e}^{x}$ and found its inverse. Their solution is recognized as the Lambert function, whose contemporary notation is $W$ and, therefore, the inverse of $y=x \mathrm{e}^{x}$ from Ref. [71] is given by $x=W(y)$.

- Tables Tables of mathematical properties of the Lambert $W$ function and their integrals: [84,85] $(2004,2010)$. The former study is in Russian and the latter work is from the American National Institute of Standards and Technology (NIST).

- Ph.D. Theses Linear time-delayed systems, growth models for plants, etc [86-90] (2007-2012).

- Reviews Asymptotic behaviors, links to trinomial zeros, solar cells, biochemical kinetics, enzyme catalysis, radiobiological models for radiotherapy in medicine, ecology and evolution, etc. [91-105] (1996-2018).

- Conferences A workshop marking the first 20 years of a revitalization of the Lambert function, a meeting on the Lambert function alongside some other special functions in optimization [106,107] (2016).

- Websites Exactly solvable transcendental equations, exactly solvable growth models, optimization, computer assisted research mathematics and its applications priority (CARMA), fast library for number theory (FLINT), etc [108-120] (19992017).

- Posters the main features of relevance to mathematics [121] (1996), physics and engineering with a contribution to Euler's tercentenary celebration [122] (2007).

- Computational libraries, algorithms, programs (some as open source codes in FORTRAN e.g. wapr.f and matlab wapr.m) with either high or unlimited accuracy (arprec, arblib, lamW) [123-142] (1973-2018).

- Approximate, closed formulae for the Lambert function (incorporating the asymptotic behaviors of the Lambert function), e.g. a global approximate formula (a single expression with five adjusted parameters) as a rational function with very good accuracy, or alternatively, a highly accurate approximation using the Padé rational polynomials for the Lambert function [143-146] (1998-2017).

- Articles by Wright Linear and non-linear difference-differential equations, solutions of transcendental equations, etc. [147-149] (1949-1059).

- Articles by Siewert et al. Kepler's problem, Riemann's problem, critical conditions, the exact solutions of transcendental equations in mathematics and physics, etc. [150-164] (1972-1981).

- Articles by Corless et al. Lambert's $W$ function in Maple, the exact solutions of transcendental equations in mathematics and physics, delayed differential equations, etc. [165-177] (1993-2012). 
- Articles by Scott et al. Molecular physics (exchange forces for $\mathrm{H}_{2}^{+}$), general relativity, quantum mechanics, etc. [178-184] (1993-2012).

- Applications in mathematics Solutions to Riemann's problems for transcendental equations, Siewert-Burniston's method and its generalization for determining zeros of analytic functions, generalized Gaussian noise model, stiff differential equations, infinite exponentials, series of exponential equations, etc. [185-213] (1952-2018).

- Applications to systems with delayed dynamics Stability of delayed systems with repeated poles, delayed fractional-order dynamic systems, communication networks, multiple delays in synchronization phenomena, bifurcation analysis, characteristic roots of time-delay systems, eigenvalue assignment for control in time-delay systems, time-delayed response of smart material actuator under alternating electric potential, etc. [214-225] (2002-2015).

- Applications in physics Corrections in counting detectors, atomic physics (helium eigenfunctions), molecular physics, black-body radiation, quantum statistics, nonideal diodes in solid-state physics, electromagnetism, accelerator-based physics (particle storage rings), plasma physics, transport physics (the Fokker-Planck equation), laser physics, thermoelectrics, pair (positron-electron) creation in strong fields, scattering physics, nuclear magnetic resonance (NMR) physics, algorithmic aspects of the Lambert function for problems in physics, a quantummechanical Schrödinger eigen-problem with a potential in the form of the Lambert $W$ function having the exact solution via the confluent hypergeometric function (this potential is of short range and it supports a finite number of bound states), motions of projectiles in media with resistance forces, etc. [226-251] (1980-2016).

- Applications in astrophysics Solar winds, solar cells, parametrization of solar photovoltaic system, etc. [252-259] (2004-2016).

- Applications in chemistry Michaelis-Menten enzyme kinetics, NMR for biochemistry, etc. [260-276] (1997-2017).

- Applications in biomedicine Epidemics, periodic breathing in chronic heart failure, dark adaptation and the retinoid cycle of vision, infection dynamics, associations/dissociation rate constants of interacting biomolecules, statistical analysis and spatial interpolation in functional magnetic resonance imaging, acidity in solid tumor growth and invasion, a glucose-insulin dynamic system, blood oxygenation level dependent (BOLD) signals from brain temperature maps, survival of irradiated cells, etc. [277-288] (2000-2015).

- Applications in ecology and evolution Euler-Lotka equation, Lotka-Volterra equation, etc. [80,103] (2009, 2016).

- Applications in hydraulics (fluid dynamics) Flow friction, full bore pipe flow within the Colebrook-White equation, etc. [289-293] (2007-2018).

- Applications in energetics and agriculture Moisture content in transformer oil [294] (2013).

- Applications in economy Economic order quality: [295] (2012).

- Applications in sociology Spread of social phenomena (behaviors, ideas, products), explosive contagion model [296] (2016).

- Use of the Lambert function in education Complementing elementary functions by the Lambert function, the Lambert function in the introduction to intermediate 
physics, the utility of the Lambert function in chemical kinetics, undergraduate theoretical physics eduction, Wien's displacement law, quantum square well, hanging chain and the gravitational force, etc. [297-313] (2002-2018).

\subsection{Applications using trinomial roots}

Trinomial roots attracted a wide interest of researchers over a period longer than 250 years with many interesting and important applications [1-52]. In an application of the presently obtained formulae, we will give an example dealing with trinomial roots encountered in radiobiological models for radiotherapy. This illustration concerns cell survival after irradiation for which the measured data from synchronous cell populations ought to be corrected for genome multiplicity [314,315] prior to appropriate comparisons with the predictions of radiobiological models. Specifically, regarding all but the $\mathrm{G}_{1}$ phase cell populations, the corrections of the measured colony surviving fractions $F(D)$ at each dose $D$ need to be made for replications of deoxyribonucleic acid (DNA) molecules, that are the principal radiation target. Such a type of corrections yields a fractional trinomial equation with the sought single cell surviving fraction $S(D)$ raised to power $n$ where $1 \leq n \leq 2$. The resulting trinomial roots $S(D)$, amenable to proper comparisons with radiobiological models, are given by a concise analytical formula as the confluent Fox-Wright function ${ }_{1} \Psi_{1}$. The results are numerically illustrated on synthesized cell surviving fractions highlighting the competitive roles of genome multiplicity and radiation damage repair as the two components of shoulders in dose-response curves. Our analytical solutions for trinomial roots can also be applied to many other problems, including those with integer powers encountered in e.g. spatially-dependent cell surviving fractions that need to be reconstructed from the measured positron emission densities in image-guided radiotherapy [316].

\section{The complete Bell polynomials}

The multi-variate complete Bell polynomials (the exponential polynomials) [53], denoted by $B \equiv B_{k}\left(x_{1}, \ldots, x_{k}\right)$, are given by [63]:

$$
\exp \left(\sum_{m=1}^{\infty} x_{m} \frac{t^{m}}{m !}\right)=\sum_{k=0}^{\infty} B_{k}\left(x_{1}, \ldots, x_{k}\right) \frac{t^{k}}{k !}
$$

where $t$ is a parameter and $\left\{x_{1}, \ldots, x_{k}\right\}$ is a set of $k$ variables. Hereafter, all the parameters and variables are generally taken to be complex quantities. The polynomial $B_{n}$ are known explicitly through the multiple sum [63]:

$$
B_{n}\left(x_{1}, x_{2}, \ldots, x_{n}\right)=\sum_{m_{1}, \ldots, m_{k} \geq 0} \frac{n !}{m_{1} ! m_{2} ! \ldots m_{k} !}\left(\frac{x_{1}}{1 !}\right)^{m_{1}}\left(\frac{x_{2}}{2 !}\right)^{m_{2}} \ldots\left(\frac{x_{k}}{k !}\right)^{m_{k}}
$$


where the indices $\left\{m_{1}, m_{2}, \ldots\right\}$ must fulfill the single condition:

$$
m_{1}+2 m_{2}+3 m_{3}+\cdots+n m_{n}=n
$$

A recursion for $\left\{B_{k}\right\}$ can be deduced from the known exponentiation of a power series:

$$
\exp \left(\sum_{m=1}^{\infty} \alpha_{m} t^{m}\right)=\sum_{k=0}^{\infty} \beta_{k} t^{k}
$$

where $\left\{\alpha_{m}\right\}$ is known and $\left\{\beta_{k}\right\}$ is defined recursively by the relation:

$$
\beta_{k}=\frac{1}{k} \sum_{m=1}^{k} m \alpha_{m} \beta_{k-m}, \quad \beta_{0}=1
$$

Introducing the $m$ th variable $x_{m}$ by $m ! \alpha_{m}$, we can cast (2.4) into the following form:

$$
\exp \left(\sum_{m=1}^{\infty} x_{m} \frac{t^{m}}{m !}\right)=\sum_{k=0}^{\infty} y_{k} t^{k}, \quad x_{m}=m ! \alpha_{m}
$$

where $\left\{y_{k}\right\}$ is defined by a recursion deduced from (2.5) as:

$$
y_{k}=\frac{1}{k} \sum_{m=1}^{k} m \frac{x_{m}}{m !} y_{k-m}, \quad y_{0}=1
$$

Comparison of (2.4) with (2.7) leads to a relation between $B_{k}$ and $y_{k}$ :

$$
B_{k}\left(x_{1}, \ldots, x_{k}\right)=k ! y_{k}
$$

The use of this relation to replace, respectively, $y_{k}$ and $y_{k-m}$ by $B_{k}\left(x_{1}, \ldots, x_{k}\right) / k$ ! and $B_{k-m}\left(x_{1}, \ldots, x_{k-m}\right) /(k-m)$ ! in (2.7) yields the recursion for the general expansion coefficient from series (2.4):

$$
B_{k}\left(x_{1}, \ldots, x_{k}\right)=\frac{1}{k} \sum_{m=1}^{k} m\left(\begin{array}{c}
k \\
m
\end{array}\right) x_{m} B_{k-m}\left(x_{1}, \ldots, x_{k-m}\right), \quad B_{0}=1
$$

where $\left(\begin{array}{c}k \\ m\end{array}\right)$ is the binomial coefficient,

$$
\left(\begin{array}{l}
k \\
m
\end{array}\right)=\frac{k !}{m !(k-m) !} .
$$


A more general expression for the binomial coefficients $\left(\begin{array}{l}a \\ n\end{array}\right)$, where " $a$ " is not necessarily an integer, is given by:

$$
\left(\begin{array}{l}
a \\
n
\end{array}\right) \equiv \frac{\Gamma(a+1)}{n ! \Gamma(a-n+1)}=(-1)^{n} \frac{(-a)_{n}}{n !} .
$$

Here, $\Gamma$ is the gamma function which for a non-negative integer $n$ reduces to a factorial via $\Gamma(n+1)=n !(n=0,1,2, \ldots)$. Further, the quantity $(a)_{n}$ is the Pochhammer symbol (also called the rising factorial):

$$
(a)_{n}=a(a+1) \cdots(a+n-1)=\frac{\Gamma(a+n)}{\Gamma(a)},
$$

which has the following property,

$$
(a)_{n-k}=(-1)^{k} \frac{n !}{(a-n-1)_{k}} \text {. }
$$

There is also a falling factorial denoted by $[a]_{n}$, which is for any value of " $a$ " defined by:

$$
[a]_{n}=a(a-1) \cdots(a-n+1), \quad[0]_{n}=0 \quad(n \geq 1), \quad[1]_{n}=\delta_{n, 1},
$$

where $\delta_{n, m}$ is the Kronecker $\delta$-symbol,

$$
\delta_{m, m}= \begin{cases}1, & n=m \\ 0, & n \neq m\end{cases}
$$

The binomial coefficient $\left(\begin{array}{l}a \\ n\end{array}\right)$ is related to the falling factorial $[a]_{n}$ via:

$$
[a]_{n}=n !\left(\begin{array}{l}
a \\
n
\end{array}\right)
$$

Moreover, the rising and the falling factorials are connected by the expression:

$$
[a]_{n}=(-1)^{n}(-a)_{n} .
$$

We used (2.9) to calculate several Bell polynomials with the results:

$$
\begin{aligned}
B_{0}= & 1, \quad B_{1}=x_{1}, \quad B_{2}=x_{1}^{2}+x_{2}, \quad B_{3}=x_{1}^{3}+3 x_{1} x_{2}+x_{3}, \\
B_{4}= & x_{1}^{4}+6 x_{1}^{2} x_{3}+4 x_{1} x_{2}+3 x_{2}^{2}+x_{4}, \\
B_{5}= & x_{1}^{5}+10 x_{1}^{3} x_{2}+10 x_{1}^{2} x_{3}+15 x_{1} x_{2}^{2}+5 x_{1} x_{4}+10 x_{2} x_{3}+x_{5}, \\
B_{6}= & x_{1}^{6}+15 x_{1}^{4} x_{2}+20 x_{1}^{3} x_{3}+45 x_{1}^{2} x_{2}^{2}+15 x_{1}^{2} x_{4}+60 x_{1} x_{2} x_{3} \\
& +6 x_{1} x_{5}+15 x_{2}^{3}+15 x_{2} x_{4}+10 x_{3}^{2}+x_{6}, \\
B_{7}= & x_{1}^{7}+21 x_{1}^{5} x_{2}+35 x_{1}^{4} x_{3}+105 x_{1}^{3} x_{2}^{2}+35 x_{1}^{3} x_{4}
\end{aligned}
$$




$$
\begin{aligned}
& +210 x_{1}^{2} x_{2} x_{3}+21 x_{1}^{2} x_{5}+105 x_{1} x_{2}^{3}+105 x_{1} x_{2} x_{4}+70 x_{1} x_{3}^{2} \\
& +7 x_{1} x_{6}+105 x_{2}^{2} x_{3}+21 x_{2} x_{5}+35 x_{3} x_{4}+x_{7} \text {, } \\
& B_{8}=x_{1}^{8}+28 x_{1}^{6} x_{2}+56 x_{1}^{5} x_{3}+210 x_{1}^{4} x_{2}^{2}+70 x_{1}^{4} x_{4} \\
& +560 x_{1}^{3} x_{2} x_{3}+56 x_{1}^{3} x_{5}+420 x_{1}^{2} x_{2}^{3}+420 x_{1}^{2} x_{2} x_{4} \\
& +28 x_{1}^{2} x_{6}+840 x_{1} x_{2}^{2} x_{3}+168 x_{1} x_{2} x_{5}+105 x_{2}^{4}+210 x_{2}^{2} x_{4} \\
& +280 x_{2} x_{3}^{2}+28 x_{2} x_{6}+280 x_{1}^{2} x_{3}^{2}+280 x_{1} x_{3} x_{4} \\
& +56 x_{3} x_{5}+35 x_{4}^{2}+8 x_{1} x_{7}+x_{8} \text {, } \\
& B_{9}=x_{1}^{9}+36 x_{1}^{7} x_{2}+84 x_{1}^{6} x_{3}+378 x_{1}^{5} x_{2}^{2}+126 x_{1}^{5} x_{4} \\
& +1260 x_{1}^{4} x_{2} x_{3}+126 x_{1}^{4} x_{5}+1260 x_{1}^{3} x_{2}^{3}+1260 x_{1}^{3} x_{2} x_{4} \\
& +84 x_{1}^{3} x_{6}+3780 x_{1}^{2} x_{2}^{2} x_{3}+756 x_{1}^{2} x_{2} x_{5}+954 x_{1} x_{2}^{4} \\
& +1890 x_{1} x_{2}^{2} x_{4}+2520 x_{1} x_{2} x_{3}^{2}+252 x_{1} x_{2} x_{6}+840 x_{1}^{3} x_{3}^{2} \\
& +1260 x_{1}^{2} x_{3} x_{4}+504 x_{1} x_{3} x_{5}+315 x_{1} x_{4}^{2}+36 x_{1}^{2} x_{7}+9 x_{1} x_{8} \text {, } \\
& +1260 x_{2}^{3} x_{3}+378 x_{2}^{2} x_{5}+1260 x_{2} x_{3} x_{4}+36 x_{2} x_{7}+280 x_{3}^{3} \text {, } \\
& +84 x_{3} x_{6}+126 x_{4} x_{5}+x_{9} \text {, } \\
& B_{10}=x_{1}^{10}+45 x_{1}^{8} x_{2}+120 x_{1}^{7} x_{3}+630 x_{1}^{6} x_{2}^{2}+210 x_{1}^{6} x_{4}+2520 x_{1}^{5} x_{2} x_{3} \\
& +252 x_{1}^{5} x_{5}+3150 x_{1}^{4} x_{2}^{3}+3150 x_{1}^{4} x_{2} x_{4}+210 x_{1}^{4} x_{6}+12600 x_{1}^{3} x_{2}^{2} x_{3} \\
& +2520 x_{1}^{3} x_{2} x_{5}+4725 x_{1}^{2} x_{2}^{4}+9450 x_{1}^{2} x_{2}^{2} x_{4}+12600 x_{1}^{2} x_{2} x_{3}^{2} \\
& +1260 x_{1}^{2} x_{2} x_{6}+2100 x_{1}^{4} x_{3}^{2}+4200 x_{1}^{3} x_{3} x_{4}+2520 x_{1}^{2} x_{3} x_{5} \\
& +1575 x_{1}^{2} x_{4}^{2}+120 x_{1}^{3} x_{7}+45 x_{1}^{2} x_{8}+12600 x_{1} x_{2}^{3} x_{3}+3780 x_{1} x_{2}^{2} x_{5} \\
& +12600 x_{1} x_{2} x_{3} x_{4}+360 x_{1} x_{2} x_{7}+2800 x_{1} x_{3}^{3}+840 x_{1} x_{3} x_{6} \\
& +1260 x_{1} x_{4} x_{5}+10 x_{1} x_{9}+945 x_{2}^{5}+3150 x_{2}^{3} x_{4}+6300 x_{2}^{2} x_{3}^{2} \\
& +630 x_{2}^{2} x_{6}+2520 x_{2} x_{3} x_{5}+1575 x_{2} x_{4}^{2}+2100 x_{3}^{2} x_{4}+120 x_{3} x_{7} \\
& +210 x_{4} x_{6}+126 x_{5}^{2}+45 x_{2} x_{8}+x_{10} \text {. }
\end{aligned}
$$

\section{The cyclic indicator polynomials}

The multi-variate cyclic indicator polynomials $C_{k}\left(x_{1}, \ldots, x_{k}\right)$ are defined as [63]:

$$
\exp \left(\sum_{m=1}^{\infty} x_{m} \frac{t^{m}}{m}\right)=\sum_{k=0}^{\infty} C_{k}\left(x_{1}, \ldots, x_{k}\right) \frac{t^{k}}{k !}
$$

The alternative, explicit form of $C_{k}\left(x_{1}, \ldots, x_{k}\right)$ is [63]:

$$
C_{n}\left(x_{1}, x_{2}, \ldots, x_{n}\right)=\sum_{m_{1}, \ldots, m_{k} \geq 0} \frac{n !}{m_{1} ! m_{2} ! \ldots m_{k} !}\left(\frac{x_{1}}{1}\right)^{m_{1}}\left(\frac{x_{2}}{2}\right)^{m_{2}} \ldots\left(\frac{x_{k}}{k}\right)^{m_{k}}
$$


under the same condition (2.3). It is also possible to calculate $C_{k}\left(x_{1}, \ldots, x_{k}\right)$ recursively [63]:

$$
C_{k+1}\left(x_{1}, \ldots, x_{k+1}\right)=\sum_{m=0}^{k}\left(\begin{array}{l}
k \\
m
\end{array}\right) x_{m+1} C_{k-m}\left(x_{1}, \ldots, x_{k-m}\right), \quad C_{0}=1 .
$$

Comparing now (2.1) and (3.1), or (2.4) and (3.2), we can see that $C_{k}\left(x_{1}, \ldots, x_{k}\right)$ is linked to $B_{k}\left(x_{1}, \ldots, x_{k}\right)$ by the relation:

$$
C_{k}\left(x_{1}, \ldots, x_{k}\right)=B_{k}\left(y_{1}, \ldots, y_{k}\right), \quad y_{k}=(k-1) ! x_{k} .
$$

Using the results for $B_{n}$ from (2.18)-(2.25), we calculated the first eleven cyclic indicator polynomials $\left\{C_{n}\right\}$ and found some typographic errors in Riordan's book [63, p. 84]: his variables $t_{k}$ corresponds to ours $x_{k}$ and in $C_{9}$ the following 3 terms $378 t_{2}^{5} t_{2}^{2}, 3024 t^{4} t^{5}, 25920 t_{1}^{2} t$ should read as $378 t_{1}^{5} t_{2}^{2}, 3024 t_{1}^{4} t_{5}, 25920 t_{1}^{2} t_{7}$, respectively.

\section{The partial Bell polynomials}

Besides the multi-variate complete Bell polynomials $B_{n}$, there are also the multivariate partial Bell polynomials $B_{n, k}$ introduced via [63]:

$$
\mathrm{e}^{-y f(x)} D_{x}^{n} \mathrm{e}^{y f(x)}=\sum_{k=1}^{n} B_{n, k}\left(f_{1}, \ldots, f_{n-k+1}\right) y^{k}
$$

where

$$
f_{n}=D_{x}^{n} f(x), \quad f_{n} \equiv f_{n}(x), \quad D_{x}=\frac{\mathrm{d}}{\mathrm{d} x} .
$$

The explicit expression for the partial polynomial $B_{n, k}$ is given by:

$$
B_{n, k}\left(x_{1}, \ldots, x_{n-k+1}\right)=\sum_{m_{1}, \ldots, m_{k} \geq 0} \frac{n !}{m_{1} ! \ldots m_{k} !}\left(\frac{x_{1}}{1 !}\right)^{m_{1}} \ldots\left(\frac{x_{k}}{k !}\right)^{m_{k}}
$$

where the multiple sums are to be carried out over all the indices $\left\{m_{1}, m_{2}, \ldots\right\}$ that, unlike (2.3), must simultaneously satisfy two conditions

$$
\left.\begin{array}{l}
m_{1}+m_{2}+m_{3}+\cdots+m_{k}=k \\
m_{1}+2 m_{2}+3 m_{3}+\cdots+n m_{n}=n
\end{array}\right\} .
$$


Similarly to (2.9) for $\left\{B_{n}\right\}$, there is also the following recursion for $\left\{B_{n, k}\right\}$ :

$$
B_{n, k}\left(x_{1}, \ldots, x_{n-k+1}\right)=\sum_{m=1}^{n-k+1}\left(\begin{array}{c}
n-1 \\
m-1
\end{array}\right) x_{m} B_{n-m, k-1}\left(x_{1}, \ldots, x_{n-m-k+2}\right)
$$

with the initialization $B_{0,0}=1$. Using (4.4), it follows:

$$
\begin{aligned}
\left(\frac{f_{1}}{1 !}\right)^{m_{1}} \ldots\left(\frac{f_{k}}{k !}\right)^{m_{k}} & =\left(\frac{f_{1}}{1 ! f}\right)^{m_{1}} \ldots\left(\frac{f_{k}}{k ! f}\right)^{m_{k}} f^{m_{1}+\cdots+m_{k}}(x) \\
& =\left(\frac{f_{1}}{1 ! f}\right)^{m_{1}} \cdots\left(\frac{f_{k}}{k ! f}\right)^{m_{k}} f^{k}(x)
\end{aligned}
$$

and, thus

$$
\left(\frac{f_{1}}{1 !}\right)^{m_{1}} \ldots\left(\frac{f_{k}}{k !}\right)^{m_{k}}=\left(\frac{h_{1}}{1 !}\right)^{m_{1}} \ldots\left(\frac{h_{k}}{k !}\right)^{m_{k}} f^{k}(x)
$$

where

$$
h_{n}=\frac{f_{n}}{f(x)}, \quad h_{n} \equiv h_{n}(x)
$$

This implies the scaling:

$$
B_{n, k}\left(f_{1}, \ldots, f_{n-k+1}\right)=f^{k}(x) B_{n, k}\left(h_{1}, \ldots, h_{n-k+1}\right) .
$$

Therefore, (4.1) can also be given by:

$$
\mathrm{e}^{-y f(x)} D_{x}^{n} \mathrm{e}^{y f(x)}=\sum_{k=1}^{n} B_{n, k}\left(h_{1}, \ldots, h_{n-k+1}\right) y^{k} f^{k}(x) .
$$

We can set $y=1$ in (4.9) and then substitute $B_{n, k}\left(f_{1}, \ldots, f_{n-k+1}\right)$ for $f^{k}(x) B_{n, k}\left(h_{1}, \ldots, h_{n-k+1}\right)$, as per (4.8). In such a case, (4.9) becomes:

$$
\mathrm{e}^{-f(x)} D_{x}^{n} \mathrm{e}^{f(x)}=\sum_{k=1}^{n} B_{n, k}\left(f_{1}, \ldots, f_{n-k+1}\right)
$$

On the other hand, we have:

$$
B_{n}\left(f_{1}, \ldots, f_{n}\right)=\sum_{k=1}^{n} B_{n, k}\left(f_{1}, \ldots, f_{n-k+1}\right)
$$

and this simplifies (4.10) as follows [53]

$$
\mathrm{e}^{-f(x)} D_{x}^{n} \mathrm{e}^{f(x)}=B_{n}\left(f_{1}, \ldots, f_{n}\right) .
$$


We have extracted the first few polynomials $B_{n, k}\left(f_{1}, \ldots, f_{n-k+1}\right)$ from the definition (4.3) and they read as:

$$
B_{1,1}=x_{1}, B_{2,1}=x_{2}, B_{2,2}=x_{1}^{2}, B_{3,1}=x_{3}, B_{3,2}=3 x_{1} x_{2}, B_{3,3}=x_{1}^{3}
$$

An extended table of $B_{n . k}$ with $1 \leq k \leq n \leq 12$ can be found in Ref. [317].

\section{Derivatives of any analytical function raised to an arbitrary power}

As a digression, the justification of which will be given in the subsequent analysis, we are now looking for the $n$th derivative of the function $1 / f^{\lambda}(x)$ :

$$
\left.R_{n, \lambda}(x) \equiv D_{x}^{n} \frac{1}{f^{\lambda}(x)}\right)=\left(\frac{\mathrm{d}}{\mathrm{d} x}\right)^{n} \frac{1}{f^{\lambda}(x)}
$$

where $\lambda$ is an arbitrary parameter (real or complex) and $f(x)$ is any analytical function. We start from the following integral representation of $1 / f^{\lambda}(x)$ :

$$
\frac{1}{f^{\lambda}(x)}=\frac{1}{\Gamma(\lambda)} \int_{0}^{\infty} \mathrm{d} u u^{\lambda-1} \mathrm{e}^{-u f(x)}
$$

Inserting (5.2) into (5.1) yields the intermediate integral:

$$
R_{n, \lambda}(x)=\frac{1}{\Gamma(\lambda)} \int_{0}^{\infty} \mathrm{d} u u^{\lambda-1} \mathrm{e}^{-u f(x)}\left\{\mathrm{e}^{u f(x)} D_{x}^{n} \mathrm{e}^{-u f(x)}\right\}
$$

The expression in the curly brackets is of the type of the lhs Eq. (4.1) and this gives:

$$
R_{n, \lambda}(x)=\sum_{k=1}^{n} B_{n, k}\left(h_{1}, \ldots, h_{n-k+1}\right)\{-f(x)\}^{k}\left\{\frac{1}{\Gamma(\lambda)} \int_{0}^{\infty} \mathrm{d} u u^{\lambda+k-1} \mathrm{e}^{-u f(x)}\right\}
$$

The result of the integral in the curly brackets in (5.4) can be obtained employing (5.2):

$$
\frac{1}{\Gamma(\lambda)} \int_{0}^{\infty} \mathrm{d} u u^{\lambda+k-1} \mathrm{e}^{-u f(x)}=\frac{\Gamma(\lambda+k)}{\Gamma(\lambda)} \frac{1}{f^{\lambda+k}(x)} .
$$


Here, we use (5.3) to identify the term $\Gamma(\lambda+k) / \Gamma(\lambda)$ as the Pochhammer symbol $(\lambda)_{k}$ as per (2.12). Then, inserting (5.5) into (5.4), we have:

$$
\left(\frac{\mathrm{d}}{\mathrm{d} x}\right)^{n} \frac{1}{f^{\lambda}(x)}=\frac{1}{f^{\lambda}(x)} \sum_{k=1}^{n}(-1)^{k}(\lambda)_{k} B_{n, k}\left(h_{1}, \ldots, h_{n-k+1}\right) .
$$

The sum over $k$ in (5.6) can be carried out by using the following relationship which connects the partial and complete Bell polynomials:

$$
\sum_{k=1}^{n}(-1)^{k}(\lambda)_{k} B_{n, k}\left(h_{1}, \ldots, h_{n-k+1}\right)=B_{n}\left(\zeta f_{1}, \ldots, \zeta f_{n}\right)
$$

with

$$
\zeta^{m}=\frac{(-1)^{m}(\lambda)_{m}}{f^{m}(x)}
$$

where $f_{m}(x)$ is given by (4.2). Finally, the $n$th derivative of function $1 / f^{\lambda}(x)$ becomes:

$$
\left(\frac{\mathrm{d}}{\mathrm{d} x}\right)^{n} \frac{1}{f^{\lambda}(x)}=\frac{B_{n}\left(\zeta f_{1}, \ldots, \zeta f_{n}\right)}{f^{\lambda}(x)}
$$

\section{An arbitrary power of a MacLaurin series of any function}

Here, we specify the general function $f(x)$ from the preceding section to be given by its MacLaurin series:

$$
f(x)=\sum_{n=0}^{\infty} a_{n} x^{n}
$$

where the elements of the set $\left\{a_{n}\right\}$ are the expansion coefficients. We are interested in obtaining the result for an arbitrary power of the MacLaurin series in (6.1) which, as an analytical function, is differentiable any number of times. By definition, the MacLaurin series of any analytical function $1 / f^{\lambda}(x)$ reads as:

$$
\frac{1}{f^{\lambda}(x)}=\sum_{n=0}^{\infty} b_{n} \frac{x^{n}}{n !}
$$

where the general expansion coefficient $b_{n}$ is:

$$
b_{n}=\left\{D_{x}^{n} \frac{1}{f^{\lambda}(x)}\right\}_{x=0} .
$$


This is the justification for considering the $n$th derivative of $1 / f^{\lambda}(x)$ in the preceding section. The reason for investigating an arbitrary power of a series expansion in the first place is dictated by the method of finding the trinomial roots in the form of a series. For $f(x)$ given by the series (6.1), it follows:

$$
f_{n}(x)=D_{x}^{n} f(x)=D_{x}^{n} \sum_{m=0}^{\infty} a_{m} x^{m}=\sum_{m=n}^{\infty}(-1)^{m}(-m)_{n} a_{m} x^{m-n},
$$

so that

$$
f^{\lambda}(0)=a_{0}^{-\lambda}, \quad f_{n}(0)=n ! a_{n} .
$$

With (6.5) at hand, the coefficient $b_{n}$ becomes:

$$
b_{n}=B_{n}\left(\xi f_{1}, \ldots, \xi f_{n}\right),
$$

where

$$
\xi^{m}=\frac{(-1)^{m}(\lambda)_{m}}{a_{0}^{\lambda+m}}
$$

Hence, an arbitrary power of the MacLaurin series (6.6) is compactly written as:

$$
\left(\sum_{n=0}^{\infty} a_{n} x^{n}\right)^{-\lambda}=\sum_{n=0}^{\infty} B_{n}\left(\xi a_{1}, \ldots, \xi a_{n}\right) \frac{x^{n}}{n !}
$$

The two values of $\lambda$ are of special interest. First, the case $\lambda=1$ is for reversion of a series when (6.8) reduces to:

$$
\frac{1}{\sum_{n=0}^{\infty} a_{n} x^{n}}=\sum_{n=0}^{\infty} B_{n}\left(\xi a_{1}, \ldots, \xi a_{n}\right) \frac{x^{n}}{n !}, \quad \xi^{k}=\frac{(-1)^{k} k !}{a_{0}^{k+1}}
$$

The second case is when $\lambda$ is a negative integer $m(m=-1,-2, \ldots)$ for which $(6.8)$ becomes:

$$
\left(\sum_{n=0}^{\infty} a_{n} x^{n}\right)^{m}=\sum_{n=0}^{\infty} B_{n}\left(\xi a_{1}, \ldots, \xi a_{n}\right) \frac{x^{n}}{n !}, \quad \xi^{k}=[m]_{k} a_{0}^{m-k}
$$




\section{The Lambert series solution for all the roots of trinomial equations}

The Euler $T(x)$ and the Lambert $W(x)$ functions are defined as the solutions of the following transcendental equations:

$$
\begin{aligned}
& y=x \mathrm{e}^{-x} \quad \therefore \quad x=T(y), \\
& y=x \mathrm{e}^{x} \quad \therefore \quad x=W(y) .
\end{aligned}
$$

The replacement of $x$ by $T(y)$ in (7.1) and $x$ by $W(y)$ in (7.2) yields the equivalent definitions of the Euler and Lambert functions:

$$
\begin{aligned}
& y=T(y) \mathrm{e}^{-T(y)}, \\
& y=W(y) \mathrm{e}^{W(y)} .
\end{aligned}
$$

Alternative to the linear-exponential forms (7.3) and (7.4), the $T$ and $W$ functions can be introduced through the linear-logarithmic relationships. Namely, taking the natural logarithm of both sides of Eq. (7.3), it follows:

$$
\begin{aligned}
\ln T(y)-T(y) & =\ln y, \\
\ln W(y)+W(y) & =\ln y .
\end{aligned}
$$

Among several representations of these functions, the power series expansions are given in the explicit forms:

$$
\begin{aligned}
& T(y) \equiv \sum_{n=1}^{\infty} \frac{n^{n-1}}{n !} y^{n}=y+y^{2}+\frac{3}{2} y^{3}+\frac{8}{3} y^{4}+\frac{125}{24} y^{5}+\frac{54}{5} y^{6}+\frac{16807}{720} y^{7}+\cdots \\
& W(y) \equiv \sum_{n=1}^{\infty} \frac{(-n)^{n-1}}{n !} y^{n}=y-y^{2}+\frac{3}{2} y^{3}-\frac{8}{3} y^{4}+\frac{125}{24} y^{5}-\frac{54}{5} y^{6}+\frac{16807}{720} y^{7}-\cdots
\end{aligned}
$$

The following evident relationship between the $T$ and $W$ functions shows that neither function is odd (symmetric) nor even (asymmetric):

$$
W(-x)=-T(x)
$$

The Euler and Lambert functions have not originally appeared in the literature in the way they are usually introduced through Eqs. (7.1) or (7.3) and (7.2) or (7.4), respectively. Rather, Lambert [1] first discovered that all the roots $x$ of the trinomial equation:

$$
x=q+x^{n} \quad(n=1,2,3, \ldots)
$$


where $q$ is a fixed parameter and $n$ any positive integer, can be expressed precisely as a series of the type from the rhs of Eq. (7.8). On the other hand, the series (7.8) is obtained as the solution the transcendental equation (7.2). As such, this dualism is the origin of using the name Lambert function for all the roots $x$ via $x=W(y)$ of the implicit equation (7.2). Thus, the original function, which since the 1990s is called the Lambert $W$ function, does not stem from an explicit search of an inverse of the function $y=x \mathrm{e}^{x}$. In fact, the actual inverse $\left(x \mathrm{e}^{x}\right)^{(-1)}$ of the function $x \mathrm{e}^{x}$ has repeatedly been established by e.g. Pólya and Szegó [71] and others. However, since the same Lambert function $W$ is the common solution $x$ to the two seemingly different problems (7.2) and (7.10), it ought to be an equivalence between the two problems. This can indeed be shown by e.g. reference to the related work of Euler [4], who in his analysis of the Lambert series (7.8), re-wrote Eq. (7.10) in a symmetrized form:

$$
x^{\alpha}-x^{\beta}=(\alpha-\beta) v x^{\alpha+\beta} \quad(\alpha, \beta, v: \text { any constants })
$$

where $v, \alpha$ and $\beta$ are known. In the special case $\alpha=1$ and $\beta=n$, it follows that (7.11) is reduced to an equation of the form (7.10) as given by:

$$
\tilde{x}=q-\tilde{x}^{n}, \quad q=v(n-1), \quad \tilde{x}=\frac{1}{x} \quad(x \neq 0) .
$$

For $\alpha \neq \beta$, both sides of Eq. (7.11) can be divided by $\alpha-\beta$ in which case the lhs of the ensuing equation $\left(x^{\alpha}-x^{\beta}\right) /(\alpha-\beta)=v x^{\alpha+\beta}$ would become an undetermined expression $0 / 0$ in the limit $\beta \rightarrow \alpha$. Then, l'Hôpital's rule would give:

$$
\lim _{\beta \rightarrow \alpha} v x^{\alpha+\beta}=v x^{2 \alpha}=\lim _{\beta \rightarrow \alpha} \frac{x^{\alpha}-x^{\beta}}{\alpha-\beta}=\lim _{\beta \rightarrow \alpha} \frac{\mathrm{d}}{\mathrm{d} \beta} \frac{x^{\alpha}-x^{\beta}}{\alpha-\beta}=x^{\alpha} \ln x,
$$

so that

$$
\ln x=v x^{\alpha}
$$

To find the solution of (7.14), we first change $x$ to $X$ via:

$$
x=\mathrm{e}^{X},
$$

and this gives

$$
X=v \mathrm{e}^{\alpha X} .
$$

Multiplying both sides of this equation by $\alpha \mathrm{e}^{-\alpha X}$ yields:

$$
Y \mathrm{e}^{-Y}=v \alpha, \quad Y=\alpha X
$$


By virtue of the relation (7.1) for the Euler $T$ function, it follows from (7.17) that:

$$
Y=T(v \alpha)
$$

Returning to the original variable $x$ via $Y=\alpha X$, where $X=\ln x$, according to (7.15), we finally have:

$$
\ln x=\frac{1}{\alpha} T(v \alpha) .
$$

Thus the closed form solution for all the roots $x$ of trinomial characteristic equation (7.14) is:

$$
x=\mathrm{e}^{(1 / \alpha) T(v \alpha)},
$$

where $T(y)$ is given by the rhs of (7.7), which Euler [4] calls the Lambert series. This derivation is based upon the definition (7.3) for the $T$ function. Another derivation could also be carried out by exploiting the fact that both the problem (7.14) and the alternative definition (7.5) for the $T$ function contain a logarithmic function. Thus, we first multiply both sides of Eq. (7.5) by $\alpha$ to write $v \alpha x^{\alpha}=\alpha \ln x$, or equivalently, $v \alpha x^{\alpha}=\ln x^{\alpha}$. Then, we add the term $\ln v \alpha$ to both sides of this latter equation, to write $v \alpha x^{\alpha}+\ln v \alpha=\ln x^{\alpha}+\ln v \alpha=\ln v \alpha x^{\alpha}$, so that after rearranging, we obtain:

$$
\ln Z-Z=\ln v \alpha
$$

where,

$$
Z=v \alpha x^{\alpha}
$$

Comparison between (7.5) and (7.21) leads to the identification:

$$
Z=T(v \alpha)
$$

Returning to the original variable by means of (7.23) yields the final result for $x$ raised to the power $\alpha$ as:

$$
x^{\alpha}=\frac{1}{v \alpha} T(v \alpha)
$$

Thus, this second derivation gives directly $x^{\alpha}$ in terms of the constant $v \alpha$ through the function $(v \alpha)^{-1} T(v \alpha)$. Correctness of the derivations based upon the two equivalent definitions (7.3) and (7.5) can be checked through cancellation of the common term $T(v \alpha)$ in division of (7.20) by (7.24):

$$
\frac{\ln x}{x^{\alpha}}=\frac{T(v \alpha) / \alpha}{T(v \alpha) /(v \alpha)}=v \quad \therefore \quad \frac{\ln x}{x^{\alpha}}=v \quad(\mathrm{QED}),
$$

in agreement with the initial problem $\ln x=v x^{\alpha}$ from Eq. (7.14). 
Overall, we started by searching the solution of the original Lambert [1] trinomial characteristic equation (7.10). However, already at the outset, this main problem was replaced by its symmetrized version (7.11) due to Euler [4]. Thus, instead of (7.10), we solved the related problem (7.11). Nevertheless, a similar procedure of solving (7.11) can also be adapted to (7.10), which this time we re-write in a more general form:

$$
x=q+x^{\alpha}
$$

where $\alpha$ is any real number, i.e. not necessarily an integer.

\section{All the trinomial roots in terms of the Bell polynomials}

Here, we shall address the main topic of the present study, and that is finding all the roots of the trinomial characteristic equation:

$$
x-y x^{\alpha}-1=0 \quad(\alpha: \text { any constant })
$$

where $\alpha$ and $y$ are known. Here, as in Euler's Eq. (7.11), power $\alpha$ is any constant (real, complex). In other words, unlike Lambert's Eq. (7.10), power $\alpha$ of the root $x$ in (8.1) does not need to be restricted exclusively to the set of integer numbers. In the course of the analysis, we shall present a novel method based upon the use of the Bell polynomials for raising a series for $x$ to the power $\alpha$. Note that there is no need to consider a more general trinomial equation:

$$
z^{\alpha}-\beta z+\gamma=0
$$

This is the case because (8.2) is reduced to (8.1) for $\beta \neq 0$ and $\gamma \neq 0$ by setting $x=(\gamma / \beta) z$ and $y=\gamma^{\alpha-1} \beta^{-\alpha}$.

A convenient starting point to solve (8.1) for $x$ is to develop $x$ in powers of $y$ :

$$
x=\sum_{n=0}^{\infty} b_{n} y^{n},
$$

where $\left\{b_{n}\right\}(n=1,2,3, \ldots)$ is the infinite set of the unknown expansion coefficients. To find the general coefficient $b_{n}$, we insert (8.3) into (8.1) and write:

$$
\sum_{n=0}^{\infty} b_{n} y^{n}=1+y\left(\sum_{n=0}^{\infty} b_{n} y^{n}\right)^{\alpha} .
$$

On the rhs of Eq. (8.4), the series (8.3) is raised to the power $\alpha$. It is for this reason that it was necessary to find the general formula (6.8) for a series raised to an arbitrary power. Thus, we employ (6.8) in (8.4) viz: 


$$
\left(\sum_{n=0}^{\infty} b_{n} y^{n}\right)^{\alpha}=\sum_{n=0}^{\infty} B_{n}\left(1 ! \zeta b_{1}, 2 ! \zeta b_{2}, \ldots, n ! \zeta b_{n}\right) \frac{y^{n}}{n !}
$$

where,

$$
\zeta^{k}=(-1)^{k}(-\alpha)_{k} b_{0}^{\alpha-k}
$$

Substituting now (8.5) into (8.4), it follows:

$$
\sum_{n=0}^{\infty} b_{n} y^{n}=1+y \sum_{n=0}^{\infty} B_{n}\left(1 ! \zeta b_{1}, 2 ! \zeta b_{2}, \ldots, n ! \zeta b_{n}\right) \frac{y^{n}}{n !} .
$$

Equating the coefficients of the same powers of $y$ from both sides of Eq. (8.7), we connect $b_{n}$ with $B_{n}$ as:

$$
b_{n}=\frac{B_{n-1}\left(1 ! \zeta b_{1}, 2 ! \zeta b_{2}, \ldots,(n-1) ! \zeta b_{n-1}\right)}{(n-1) !}, b_{0}=1(n>1) .
$$

This result can equivalently be given through the cyclic indicator polynomials using (3.4):

$$
b_{n}=\frac{C_{n-1}\left(\zeta b_{1}, \zeta b_{2}, \ldots, \zeta b_{n-1}\right)}{(n-1) !}, b_{0}=1(n>1) .
$$

Therefore, all the roots of the transcendental equation (8.1) are expressed either through the complete Bell polynomials:

$$
\left.\begin{array}{l}
x-y x^{\alpha}-1=0 \\
x=\sum_{n=0}^{\infty} B_{n-1}\left(1 ! \zeta b_{1}, 2 ! \zeta b_{2}, \ldots,(n-1) ! \zeta b_{n-1}\right) \frac{y^{n}}{(n-1) !}
\end{array}\right\},
$$

or through the cyclic indicator polynomials,

$$
\left.\begin{array}{l}
x-y x^{\alpha}-1=0 \\
x=\sum_{n=0}^{\infty} C_{n-1}\left(\zeta b_{1}, \zeta b_{2}, \ldots, \zeta b_{n-1}\right) \frac{y^{n}}{(n-1) !}
\end{array}\right\} .
$$

In the sums from (8.10) and (8.11), the term with $n=0$ is, by definition, equal to 1 .

\section{From multi- to uni-variate polynomials for trinomial roots}

There is more to the result (8.10) and that is a further simplification of the complete Bell polynomials. To illustrate this point, we make use of (2.18) and (2.19) to explicitly calculate the first few coefficients $b_{n}(0 \leq n \leq 4)$ as:

$$
b_{0}=1, b_{1}=1, b_{2}=\alpha, b_{3}=\frac{\alpha(3 \alpha-1)}{2 !} .
$$


With more details, the next coefficient $\left(b_{4}\right)$ is also reduced to a simple form:

$$
\begin{aligned}
3 ! b_{4} & =B_{3}\left(1 ! \zeta b_{1}, 2 ! \zeta b_{2}, 3 ! \zeta b_{3}\right) \\
& =\left(1 ! \zeta b_{1}\right)^{3}+3\left(1 ! \zeta b_{1}\right)\left(2 ! \zeta b_{2}\right)+3 ! \zeta b_{3} \\
& =\zeta^{3} b_{1}^{3}+3\left(2 ! \zeta^{2} b_{1} b_{2}\right)+3 ! \zeta b_{3} \\
& =(-1)^{3}(-\alpha)_{3}+6(-1)^{2}(-\alpha)_{2} \alpha+6(-1)^{1}(-\alpha)_{1} \frac{\alpha(3 \alpha-1)}{2} \\
& =\alpha(\alpha-1)(\alpha-2)+6 \alpha^{2}(\alpha-1)+3 \alpha^{2}(3 \alpha-1) \\
& =\alpha\left(16 \alpha^{2}-8 \alpha-4 \alpha+2\right)=16 \alpha^{3}-12 \alpha^{2}+2 \alpha
\end{aligned}
$$

so that,

$$
b_{4}=\frac{16 \alpha^{3}-12 \alpha^{2}+2 \alpha}{3 !}
$$

A similar calculation for $b_{5}$ and $b_{6}$ yields the final results:

$$
\begin{aligned}
& b_{5}=\frac{125 \alpha^{4}-150 \alpha^{3}+55 \alpha^{2}-6 \alpha}{4 !}, \\
& b_{6}=\frac{1296 \alpha^{5}-2160 \alpha^{4}+1260 \alpha^{3}-300 \alpha^{2}+24 \alpha}{5 !} .
\end{aligned}
$$

Thus, in general, $(n-1) ! b_{n}$ as is a polynomial (8.8), say $p_{n-1}(\alpha)$ of degree $n-1$ in the variable $\alpha$ with no free term and with the integer coefficients:

$$
b_{n}=\frac{p_{n-1}(\alpha)}{(n-1) !}, \quad n \geq 2 \quad\left(b_{0}=b_{1}=1\right),
$$

with

$$
\begin{aligned}
& p_{1}(\alpha)=\alpha \\
& p_{2}(\alpha)=3 \alpha^{2}-\alpha \\
& p_{3}(\alpha)=16 \alpha^{3}-12 \alpha^{2}+2 \alpha . \\
& p_{4}(\alpha)=125 \alpha^{4}-150 \alpha^{3}+55 \alpha^{2}-6 \alpha . \\
& p_{5}(\alpha)=1296 \alpha^{5}-2160 \alpha^{4}+1260 \alpha^{3}-300 \alpha^{2}+24 \alpha, \quad \text { etc. }
\end{aligned}
$$

On the other hand, according to (8.8), the same general term $(n-1) ! b_{n}=p_{n-1}(\alpha)$ is also the Bell polynomial $(n-1) ! b_{n}=B_{n-1}\left(1 ! \zeta b_{1}, 2 ! \zeta b_{2}, \ldots,(n-1) ! \zeta b_{n-1}\right)$. In such a way, the particular multi-variate Bell polynomial $B_{n-1}\left(1 ! \zeta b_{1}, 2 ! \zeta b_{2}, \ldots,(n-\right.$ $\left.1) ! \zeta b_{n-1}\right)$ in the $n$ specified variables $\left\{x_{1}, x_{2}, \ldots, x_{n}\right\}=\left\{\zeta 1 ! b_{1}, \zeta 2 ! b_{2}, \ldots, \zeta(n-\right.$ $\left.1) ! b_{n-1}\right\}$ becomes, in fact, the uni-variate polynomial $p_{n-1}(\alpha)$ in the variable $\alpha$ :

$$
B_{n}\left(1 ! \zeta b_{1}, 2 ! \zeta b_{2}, \ldots, n ! \zeta b_{n}\right)=p_{n}(\alpha)
$$


Here, the polynomial $p_{n}(\alpha)$ is the uni-variate polynomial in variable $\alpha$. Moreover, these latter polynomials can be represented in a more convenient factored form. Namely, by returning to e.g. (9.2), the line $\alpha\left(\alpha^{2}-8 \alpha-4 \alpha+2\right)$, which precedes the final result $16 \alpha^{3}-12 \alpha^{2}+2 \alpha$, can be rewritten as $\alpha\left(\alpha^{2}-8 \alpha-4 \alpha+2\right)=\alpha[4 \alpha(4 \alpha-1)-$ $2(4 \alpha-1)]=\alpha(4 \alpha-1)(4 \alpha-2)$. Therefore, $b_{4}$ from (9.3) is equivalently given by:

$$
b_{4}=\frac{\alpha(4 \alpha-1)(4 \alpha-2)}{3 !}
$$

Further, by the like reductions of the corresponding intermediate expressions within $b_{5}$ and $b_{6}$, i.e. prior to obtaining the polynomials in (9.4) and (9.5), we explicitly verified that the following is true:

$$
\begin{aligned}
& b_{5}=\frac{\alpha(5 \alpha-1)(5 \alpha-2)(5 \alpha-3)}{4 !}, \\
& b_{6}=\frac{\alpha(6 \alpha-1)(6 \alpha-2)(6 \alpha-3)(6 \alpha-4)}{5 !} .
\end{aligned}
$$

Hence, this self-evident pattern infers the following factored form of the general expansion coefficient $b_{n}$ from (8.3):

$$
\begin{aligned}
b_{n} & =\frac{\alpha(n \alpha-1)(n \alpha-2)(n \alpha-3) \cdots(n \alpha-n+2)}{(n-1) !}, \\
& =\frac{\alpha}{(n-1) !} \prod_{k=1}^{n-2}(n \alpha-k), \quad n \geq 3 \quad\left(b_{0}=b_{1}=1, \quad b_{2}=\alpha\right) .
\end{aligned}
$$

The outlined derivation simplifies the expression in (8.11) according to:

$$
\begin{aligned}
& x-y x^{\alpha}-1=0 \\
& \left.x=\sum_{n=0}^{\infty}\{\alpha(n \alpha-1)(n \alpha-2)(n \alpha-3) \cdots(n \alpha-n+2)\} \frac{y^{n}}{(n-1 !}\right\} .
\end{aligned}
$$

This finding coincides with the result of Euler [3] from 1777. Most recently, the proof of (9.17) has also been given by Wang [50] in 2016.

\section{All the trinomial roots by a series in terms of the Pochhammer symbols}

Equivalently, (9.16) can be cast into another form involving the Pochhammer symbol (2.12):

$$
b_{n}=\frac{(-1)^{n} \alpha}{(n-1) !}(1-n \alpha)_{n-2} \text {. }
$$


Referring to (9.6), we see that the Bell uni-variate polynomials $p_{n}$ acquire the following concise expression:

$$
\begin{aligned}
& p_{n}(\alpha)=\alpha \prod_{k=1}^{n-1}(n \alpha-\alpha-k) \\
& p_{n}(\alpha)=(-1)^{n+1} \alpha(1-n \alpha-\alpha)_{n-1}
\end{aligned}
$$

In particular, (10.2) is recognized as the canonical representation of $p_{n}(\alpha)$ written as the product of the monomials $\alpha-\alpha_{n, k}$ :

$$
p_{n}(\alpha)=\alpha(n+1)^{n-1} \prod_{k=1}^{n-1}\left(\alpha-\alpha_{n, k}\right)
$$

where $\left\{\alpha_{n, k}\right\}$ is the set of the roots that are all positive rational numbers smaller than unity:

$$
\alpha_{n, k}=\frac{k}{n+1}<1 \quad(1 \leq k \leq n-1)
$$

Thus, all the roots of the transcendental equation (8.1) are given by the series (8.3) with the expansion coefficients $\left\{b_{n}\right\}$ from (10.1), as summarized by:

$$
\left.\begin{array}{l}
x-y x^{\alpha}-1=0 \\
x=1+\alpha \sum_{n=1}^{\infty}(1-n \alpha)_{n-2} \frac{(-y)^{n}}{(n-1 !}
\end{array}\right\} .
$$

\section{Arbitrary real- or complex-valued powers of trinomial roots}

Regarding the roots $x$ of the transcendental equation (8.3), it is also of interest to find the power function $x^{\beta}$ where $\beta$ is any real or complex parameter. This can be done by starting from the series (8.3) to write:

$$
\begin{aligned}
x^{\beta} & =\left(\sum_{n=0}^{\infty} b_{n} y^{n}\right)^{\beta} \\
& =\sum_{n=0}^{\infty} B_{n}\left(1 ! \xi b_{1}, 2 ! \xi b_{2}, \ldots, n ! \xi b_{n}\right) \frac{y^{n}}{n !} \\
& =\sum_{n=0}^{\infty} c_{n} y^{n}
\end{aligned}
$$


where

$$
\begin{aligned}
\xi^{k} & =(-1)^{k}(-\beta)_{k} b_{0}^{\beta-k}, \\
c_{n} & =\frac{1}{n !} B_{n}\left(1 ! \xi b_{1}, 2 ! \xi b_{2}, \ldots, n ! \xi b_{n}\right) .
\end{aligned}
$$

The first few expansion coefficients $\left\{c_{n}\right\}$, are found by using the expressions (2.18)(2.25) for the Bell polynomial with the results:

$$
\begin{aligned}
c_{0}= & 1, \quad c_{1}=\beta, \quad c_{2}=\frac{\beta}{2 !}[(2 \alpha-1)+\beta] \\
3 ! c_{3}= & B_{3}\left(1 ! \xi b_{1}, 2 ! \xi b_{2}, 3 ! \xi b_{3}\right) \\
= & \left(1 ! \xi b_{1}\right)^{3}+3\left(1 ! \xi b_{1}\right)\left(2 ! \xi b_{2}\right)+3 ! \xi b_{3} \\
= & \xi^{3} b_{1}^{3}+6 \xi^{2} b_{1} b_{2}+6 \xi b_{3} \\
= & (-1)^{3}(-\beta)_{3}+6(-1)^{2}(-\beta)_{2} \alpha+6(-1)^{1}(-\beta)_{1} \frac{\alpha(3 \alpha-1)}{2} \\
= & \beta[(\beta-1)(\beta-2)+6(\beta-1) \alpha+3 \alpha(3 \alpha-1)] \\
= & \beta\{[(3 \alpha-1)(3 \alpha-2)]+[\beta(\beta-1)]+[2 \beta(3 \alpha-1)]\} \\
c_{3}= & \frac{\beta}{3 !}\{[(3 \alpha-1)(3 \alpha-2)]+[\beta(\beta-1)+2 \beta(3 \alpha-1)]\} \\
c_{4}= & \frac{\beta}{4 !}\{[(4 \alpha-1)(4 \alpha-2)(4 \alpha-3)]+[\beta(\beta-1)(\beta-2) \\
& +3 \beta(\beta-1)(4 \alpha-1)+3 \beta(4 \alpha-1)(4 \alpha-2)]\} \\
c_{5}= & \frac{\beta}{5 !}\{[(5 \alpha-1)(5 \alpha-2)(5 \alpha-3)(5 \alpha-4)]+\beta[(\beta-1)(\beta-2)(\beta-3) \\
& +4(\beta-1)(\beta-2)(5 \alpha-1)+6(\beta-1)(5 \alpha-1)(5 \alpha-2) \\
& +4(5 \alpha-1)(5 \alpha-2)(5 \alpha-3)]\}
\end{aligned}
$$

The pattern which emerges from here is clear as each $c_{n}(2 \leq n \leq 5)$ is a sum of $n$ structurally grouped terms. The structure is such that each $c_{n}$ has the three types of products, the ones involving only the parameter $\alpha$ (stemming from the expansion coefficients $\left\{b_{n}\right\}$ ), the ones with the parameter $\beta$ alone and the mixed terms having $\alpha$ as well as $\beta$. Because of such a special structure, it is possible to express every $c_{n}$ in a more compact way comprised of only $n$ product of mixed terms. For example, in the case of $c_{3}$ from (11.5), we have:

$$
\begin{aligned}
\frac{3 !}{\beta} c_{3} & =(3 \alpha-1)(3 \alpha-2)+\beta(\beta-1)+2 \beta(3 \alpha-1) \\
& =\{(3 \alpha-1)(3 \alpha-2)+\beta(3 \alpha-1)\}+\{\beta(\beta-1)+\beta(3 \alpha-1)\} \\
& =\{(3 \alpha-1)(3 \alpha-2+\beta)\}+\{\beta(3 \alpha-2+\beta\} \\
& =(3 \alpha-1+\beta)(3 \alpha-2+\beta) \\
\therefore \quad c_{3} & =\frac{3 !}{\beta}(3 \alpha-1+\beta)(3 \alpha-2+\beta) .
\end{aligned}
$$


Carrying out calculations similar to $c_{3}$, by using the intermediate steps prior to arriving at (11.7) and (11.8), we obtain the following results:

$$
\begin{aligned}
& c_{4}=\frac{4 !}{\beta}(4 \alpha-1+\beta)(4 \alpha-2+\beta)(4 \alpha-3+\beta), \\
& c_{5}=\frac{5 !}{\beta}(5 \alpha-1+\beta)(5 \alpha-2+\beta)(5 \alpha-3+\beta)(5 \alpha-4+\beta) .
\end{aligned}
$$

This evidently implies the general formula for the expansion coefficient $c_{n}$ for any subscript $n$ as:

$$
\begin{aligned}
c_{n} & =\frac{\beta}{n !}(n \alpha-1+\beta)(n \alpha-2+\beta) \cdots(n \alpha-n+1+\beta), \\
& =\frac{\beta}{n !} \prod_{k=1}^{n-1}(n \alpha-k+1+\beta),
\end{aligned}
$$

or alternatively

$$
c_{n}=\frac{(-1)^{n-1}}{n !} \beta(1-n \alpha-\beta)_{n-1}, \quad n \geq 1 \quad\left(c_{0}=1\right) .
$$

Thus, with the result (11.14) at hand, and having in mind (11.1), we can now give the power $\beta$ of the root $x$ of the transcendental equation (8.3), so that the pair of the expressions in (10.6) can be extended to add the 3rd formula containing $x^{\beta}$ :

$$
\left.\begin{array}{l}
x-y x^{\alpha}-1=0 \\
x^{\beta}=1+\beta \sum_{n=1}^{\infty}(-1)^{n-1}(1-n \alpha-\beta)_{n-1} \frac{y^{n}}{n !}
\end{array}\right\} .
$$

\section{Logarithmic function of trinomial roots}

The logarithm of the trinomial root can be found taking the limit $\beta \rightarrow 0$ in the power function $x^{\beta}$ from (11.15). First, we extract the part $\left(x^{\beta}-1\right) / \beta$ from (11.15):

$$
\frac{x^{\beta}-1}{\beta}=\sum_{n=1}^{\infty}(-1)^{n-1}(1-n \alpha-\beta)_{n-1} \frac{y^{n}}{n !} .
$$

Since the lhs of this equation is an undetermined (0/0) for $\beta \rightarrow 0$, l'Hôpital's rule applies and this generates the logarithmic function: 


$$
\begin{aligned}
\lim _{\beta \rightarrow 0} \frac{x^{\beta}-1}{\beta} & =\ln x \\
& =\lim _{\beta \rightarrow 0} \sum_{n=1}^{\infty}(-1)^{n-1}(1-n \alpha-\beta)_{n-1} \frac{y^{n}}{n !} .
\end{aligned}
$$

This gives the logarithmic function $\ln x$ of the general trinomial root $x$ as:

$$
\left.\begin{array}{l}
x-y x^{\alpha}-1=0 \\
\ln x=\sum_{n=1}^{\infty}(-1)^{n-1}(1-n \alpha)_{n-1} \frac{y^{n}}{n !}
\end{array}\right\} .
$$

\section{Trinomial roots in terms of the confluent Fox-Wright function}

First, let us examine the particular case $\beta=1$ in the power function $x^{\beta}$ from (11.15). Using (2.13), it follows for $\beta=1$ :

$$
\left\{(-1)^{n-1}(1-n \alpha-\beta)_{n-1}\right\}_{\beta=1}=(-1)^{n-1}(-n \alpha)_{n-1}=\frac{1}{n}\left(\begin{array}{c}
n \alpha \\
n-1
\end{array}\right) .
$$

Thus, for $\beta=1$ the power function $x^{\beta}$ from (11.15) is simplified to:

$$
x=1+\sum_{n=1}^{\infty}\left(\begin{array}{c}
n \alpha \\
n-1
\end{array}\right) \frac{y^{n}}{n} .
$$

Let us give an example for e.g. $\alpha=1$ for which we have $\left(\begin{array}{c}n \alpha \\ n-1\end{array}\right) / n=1$, so that (13.2) becomes $x=1+\sum_{n=1}^{\infty} y^{n}$, or equivalently, $x=\sum_{n=0}^{\infty} y^{n}$. This is correct since for $\alpha=1$, the general transcendental equation (8.1) is reduced to $x-y x-1=0$, yielding $x=1 /(1-y)=\sum_{n=0}^{\infty} y^{n}$, after using the binomial expansion of $x=1 /(1-y)$.

We can also use the definition (2.11) to express the binomial coefficient from (13.2) in terms of the gamma functions according to:

$$
\frac{1}{n}\left(\begin{array}{c}
n \alpha \\
n-1
\end{array}\right)=\frac{\Gamma(1+n \alpha)}{\Gamma(2+n\{\alpha-1\})} \frac{1}{n !}
$$

This permits expressing (13.2) in the following equivalent form:

$$
\left.\begin{array}{l}
x-y x^{\alpha}-1=0 \\
x={ }_{1} \Psi_{1}([1, \alpha] ;[2, \alpha-1] ; y)
\end{array}\right\},
$$

where ${ }_{1} \Psi_{1}$ is the confluent Fox-Wright $\Psi$-function whose general definition is given by: 


$$
{ }_{1} \Psi_{1}([a, \alpha] ;[b, \beta] ; z) \equiv \sum_{n=0}^{\infty} \frac{\Gamma(a+n \alpha)}{\Gamma(b+n \beta)} \frac{z^{n}}{n !} .
$$

The confluent Fox-Wright $\Psi$-function ${ }_{1} \Psi_{1}$ of order $(1 ; 1)$ itself is the special case of the more general Fox-Write $\Psi$-function ${ }_{n} \Psi_{m}$ of orders $(n ; m)$ :

$$
{ }_{n} \Psi_{m}\left(\left[a_{1}, \alpha_{1}\right], \ldots,\left[a_{n}, \alpha_{n}\right] ;\left[b_{1}, \beta_{1}\right], \ldots,\left[b_{m}, \beta_{m}\right] ; z\right) \equiv \sum_{k=0}^{\infty} \frac{\prod_{r=1}^{n} \Gamma\left(a_{r}+k \alpha_{r}\right)}{\prod_{s=1}^{m} \Gamma\left(b_{s}+s \beta_{s}\right)} \frac{z^{k}}{k !} .
$$

The confluent Fox-Wright function ${ }_{1} \Psi_{1}$ is an extension of the confluent Kummer hypergeometric function ${ }_{1} F_{1}$ :

$$
{ }_{1} F_{1}(a ; b ; z) \equiv \sum_{n=0}^{\infty} \frac{\Gamma(a+n)}{\Gamma(b+n)} \frac{z^{n}}{n !} .
$$

Similarly, the more involved case of the $\Psi$-function, namely ${ }_{n} \Psi_{m}$, is an extension of the generalized Gauss hypergeometric function ${ }_{n} F_{m}$ :

$$
{ }_{n} F_{m}\left(a_{1}, \ldots, a_{n} ; b_{1}, \ldots, b_{m} ; z\right) \equiv \sum_{k=0}^{\infty} \frac{\prod_{r=1}^{n} \Gamma\left(a_{r}+k\right)}{\prod_{s=1}^{m} \Gamma\left(b_{s}+s\right)} \frac{z^{k}}{k !} .
$$

Moreover, there is a complete coincidence for $a=1$ and $b=1$ in the confluent case and similarly for the general case of the two pairs of functions:

$$
\begin{aligned}
& { }_{1} \Psi_{1}([a, 1] ;[b, 1] ; z)={ }_{1} F_{1}(a ; b ; z), \\
& { }_{n} \Psi_{m}\left(\left[a_{1}, 1\right], \ldots,\left[a_{n}, 1\right] ;\left[b_{1}, 1\right], \ldots,\left[b_{m}, 1\right] ; z\right)={ }_{n} F_{m}\left(a_{1}, \ldots, a_{n} ; b_{1}, \ldots, b_{m} ; z\right) .
\end{aligned}
$$

\section{Convergence radius of the series for trinomial roots}

While the ${ }_{1} F_{1}$-function from (13.7) is convergent for every $z$ (real or complex), this is not the case for the ${ }_{1} \Psi_{1}$-function (13.5). It is, therefore, important to find the convergence radius $R$ of the series in (13.5). This latter series would converge provided that $|z|<|R|$, where:

$$
R=\lim _{n \rightarrow \infty} \frac{\gamma_{n+1}}{\gamma_{n}}, \quad \gamma_{n} \equiv \frac{\Gamma(a+n \alpha)}{\Gamma(b+n \beta)} \frac{z^{n}}{n !} .
$$

Using the well-known asymptotic form of the gamma function $\Gamma(u)$ for large values of its variable $u$ (real or complex):

$$
\Gamma(u)=\sqrt{2 \pi} u^{u-1 / 2} \mathrm{e}^{-u},
$$


it follows

$$
\gamma_{n}=\frac{(n \alpha)^{a+n \alpha}}{(n \beta)^{b+n \beta}} \mathrm{e}^{-n(\alpha-\beta)} \frac{z^{n}}{n !}
$$

so that

$$
\lim _{n \rightarrow \infty} \frac{\gamma_{n+1}}{\gamma_{n}}=\alpha^{\alpha} \beta^{-\beta} z
$$

and, therefore

$$
|R|=\left|\alpha^{\alpha} \beta^{-\beta}\right| \cdot|z|
$$

Thus, the series (13.5) for the confluent Fox-Wright ${ }_{1} \Psi_{1}$-function will converge for $|R|<1$, i.e. for $\left|\alpha^{\alpha} \beta^{-\beta}\right| \cdot|z|<1$, implying that the convergence region does not depend on the parameters $a$ and $b$ :

$$
|z|<\left|\alpha^{-\alpha} \beta^{\beta}\right|: \quad \text { Convergence region for }{ }_{1} \Psi_{1} \text { from (13.5). }
$$

Setting here $\beta=\alpha-1$ and $z=y$ regarding ${ }_{1} \Psi_{1}([1, \alpha] ;[2, \alpha-1] ; y)$ from (13.4), we see that the series for the Fox-Wright ${ }_{1} \Psi_{1}$-function representing the root $x$ of the transcendental equation (8.1) will converge for $\left|(\alpha-1)^{1-\alpha} \alpha^{\alpha}\right| \cdot|y|<1$, so that:

$$
\left.\begin{array}{l}
x-y x^{\alpha}-1=0 \\
x=1+\sum_{n=1}^{\infty}\left(\begin{array}{c}
n \alpha \\
n-1
\end{array}\right) \frac{y^{n}}{n} \\
\text { Convergence region : }|y|<\left|(\alpha-1)^{\alpha-1} \alpha^{-\alpha}\right|
\end{array}\right\} .
$$

Let us now return to (12.3) for $\ln x$ of the roots $x$ of (8.1). Therein, we can use (2.12) and (2.11) to have:

$$
(-1)^{n-1}(1-n \alpha-\beta)_{n-1} \frac{y^{n}}{n !}=\frac{\Gamma(n \alpha)}{n ! \Gamma(1+n \alpha-n)}=\frac{1}{n \alpha}\left(\begin{array}{c}
n \alpha \\
n \alpha-n
\end{array}\right) .
$$

This transformation maps (12.3) into the expression:

$$
\left.\begin{array}{l}
x-y x^{\alpha}-1=0 \\
\ln x=\frac{1}{\alpha} \sum_{n=1}^{\infty}\left(\begin{array}{c}
n \alpha \\
n \alpha-n
\end{array}\right) \frac{y^{n}}{n}
\end{array}\right\} .
$$

To find the convergence radius $\rho$ of this series, we set:

$$
\rho=\lim _{n \rightarrow \infty} \frac{\delta_{n+1}}{\delta_{n}}, \quad \delta_{n}=\frac{1}{n \alpha}\left(\begin{array}{c}
n \alpha \\
n \alpha-n
\end{array}\right)=\frac{\Gamma(n \alpha)}{n ! \Gamma(n \alpha-n+1)},
$$


and make use of (14.2) to calculate $\delta_{n+1} / \delta_{n}$ with the result:

$$
\lim _{n \rightarrow \infty} \frac{\delta_{n+1}}{\delta_{n}}=\frac{\alpha^{\alpha}}{(\alpha-1)^{\alpha-1}} y
$$

From here, the convergence requirement $|y|<|\rho|$ leads to $|y|<\left|(\alpha-1)^{\alpha-1} \alpha^{-\alpha}\right|$ so that:

$$
\left.\begin{array}{l}
x-y x^{\alpha}-1=0 \\
\ln x=\frac{1}{\alpha} \sum_{n=1}^{\infty}\left(\begin{array}{c}
n \alpha \\
n \alpha-n
\end{array}\right) \frac{y^{n}}{n} \\
\text { Convergence region : }|y|<\left|(\alpha-1)^{\alpha-1} \alpha^{-\alpha}\right|
\end{array}\right\} .
$$

Thus, the series (14.7) and (14.12) for $x$ and $\ln x$, respectively, have the same convergence radius. Note that the sum in (14.12) cannot be extended to include the term $n=0$ because in that case the numerator would be singular, $\{\Gamma(n \alpha)\}_{n=0}=\infty$.

Finally, we will consider the general case of $\beta$ arbitrary in the power function $x^{\beta}$ from (11.15). For any value of $\beta$, employing (2.13), it follows:

$$
(-1)^{n-1}(1-n \alpha-\beta)_{n-1} \frac{1}{n !}=\frac{\Gamma(n \alpha+\beta)}{n ! \Gamma(n \alpha+\beta-n+1)}=\frac{1}{n \alpha+\beta}\left(\begin{array}{c}
n \alpha+\beta \\
n
\end{array}\right),
$$

and this yields

$$
\left.\begin{array}{l}
x-y x^{\alpha}-1=0 \\
x^{\beta}=1+\beta \sum_{n=1}^{\infty}\left(\begin{array}{c}
n \alpha+\beta \\
n
\end{array}\right) \frac{y^{n}}{n \alpha+\beta}
\end{array}\right\} .
$$

Alternatively, using the gamma functions instead of the binomial coefficient, by way of (14.13), the series from (14.14) can be rewritten as:

$$
\begin{aligned}
x^{\beta}=1+\beta \sum_{n=1}^{\infty}\left(\begin{array}{c}
n \alpha+\beta \\
n
\end{array}\right) \frac{y^{n}}{n \alpha+\beta} & =1+\beta \sum_{n=1}^{\infty} \frac{\Gamma(n \alpha+\beta)}{\Gamma(n \alpha+\beta-n+1)} \frac{y^{n}}{n !} \\
& =\beta \sum_{n=0}^{\infty} \frac{\Gamma(\beta+n \alpha)}{\Gamma(\{\beta+1\}+n\{\alpha-1\})} \frac{y^{n}}{n !} .
\end{aligned}
$$

The last line in (14.15) and the definition (13.5) reduce $x^{\beta}$ the confluent Fox-Wright ${ }_{1} \Psi_{1}$-function for $x^{\beta}$ in the case of any value of the parameter $\beta$ :

$$
\left.\begin{array}{l}
x-y x^{\alpha}-1=0 \\
x^{\beta}=\beta_{1} \Psi_{1}([\beta, \alpha] ;[\beta+1, \alpha-1] ; y)
\end{array}\right\} .
$$




\section{An illustration in radiobiology for radiotherapy}

There is a large number of radiobiological models for description of cell surviving fraction $S$ after exposures to radiation by dose $D$ [101,102]. These models rely upon the two main assumptions: the critical targets of radiation are the DNA molecules and genome integrity is the prerequisite for reproduction of mammalian cells. Customarily, radiobiological models adopt an implicit assumption that no part of DNA has replicated. However this is not justified for two cases with differing types of populations in e.g. synchronized cells. One case is the cell population in the $\mathrm{S}$ (or $\mathrm{G}_{2}$ ) phase, where a fraction $n-1(1 \leq n \leq 2)$ of DNA molecules has replicated. The other case is mitotic cell population, in which a fraction $m-1(1 \leq m \leq 2)$ has a double complement of DNA due to the age spread, while the remaining fraction $(2-m)$ has divided. As such, whenever the radiation produces damages to the genome, measured surviving fractions for all but the $\mathrm{G}_{1}$ phase cell populations are affected by genome multiplicity. Thus, experimental data for the two mentioned population types should be corrected for DNA replication before making appropriate comparisons with radiobiological models that generally ignore genome multiplicity [314,315,318-330]. The pertinent corrections for both cases have been derived in Refs. [314,315] and, for the $\mathrm{S}$ (or $\mathrm{G}_{2}$ ) phase population, it follows:

$$
S^{n}(D)-2 S(D)+F(D)=0, \quad 1 \leq n \leq 2,
$$

where the dose-dependent function $F(D)$ represents the data for the measured colony surviving fractions. As such, Eq. (15.1) extracts the single cell surviving fractions $S(D)$ from the measured (observed) experimental data $F(D)$. Therefore, the solutions $S(D)$ of Eq. (15.1) are the quantities that can be compared with the single cell surviving fractions from radiobiological models. It is seen that (15.1) is an $n$th degree characteristic trinomial equation, where $n$ is not an integer.

Introducing the substitution $x=(2 / F) S$, we transform (15.1) exactly to the form of the general trinomial equation (8.1) provided that the following identification is made:

$$
\alpha=n, \quad x=2 \frac{S(D)}{F(D)}, \quad y=\frac{F^{n-1}(D)}{2^{n}} .
$$

With this at hand, and by reference to (13.4), all the solutions (roots) of the fractal trinomial equation (15.1) are given in terms of the following confluent Fox-Wright function ${ }_{1} \Psi_{1}$ with non-integer $n$ :

$$
S(D)=\frac{F(D)}{2}{ }_{1} \Psi_{1}\left([1, n] ;[2, n-1] ; \frac{F^{n-1}(D)}{2^{n}}\right), \quad 1 \leq n \leq 2 .
$$

The series representation of this formula can be extracted from (13.2) and it reads:

$$
S(D)=\frac{F(D)}{2}\left\{1+\sum_{k=1}^{\infty}\left(\begin{array}{c}
k n \\
k-1
\end{array}\right) \frac{F^{k n-k}(D)}{2^{k n} k}\right\},
$$




$$
=\frac{F(D)}{2}\left\{1+\sum_{k=1}^{\infty} \frac{\Gamma(1+k n)}{\Gamma(2+k n-k)} \frac{F^{k n-k}(D)}{2^{k n} k !}\right\},
$$

where (13.3) is used. According to (14.7), and given that $F(D) \geq 0$, the domain of validity (the convergence region) of this development expansion becomes:

$$
\frac{F^{n-1}(D)}{2^{n}}<|n-1|^{n-1} n^{-n}, \quad 1 \leq n \leq 2 .
$$

The biological effect of radiation denoted by $\mathrm{E}_{\mathrm{B}}(D)$ is defined as the negative natural logarithm of the surviving fraction, $S(D)$. Therefore, with the help of (14.12) and (15.3), it follows:

$$
\begin{aligned}
\mathrm{E}_{\mathrm{B}}(D) & \equiv-\ln S(D), \\
& =\ln \frac{2}{F(D)}-\frac{1}{n} \sum_{k=1}^{\infty}\left(\begin{array}{c}
k n \\
k n-k
\end{array}\right) \frac{F^{k n-k}(D)}{2^{k n} k}, \\
& =\ln \frac{2}{F(D)}-\sum_{k=1}^{\infty} \frac{\Gamma(k n)}{\Gamma(1+k n-k)} \frac{F^{k n-k}(D)}{2^{k n} k !},
\end{aligned}
$$

where (14.8) is employed. This series is convergent for the same values of $F(D)$ that satisfy the validity condition (15.6). Outside the convergence regions for (15.5) and (15.9), one can resum divergent series using analytical continuation by means of e.g. the Padé approximant [331].

As an illustration, we presently carried out the computations on the given synthesized cell survival fractions for two different input data that are either $S(D)$ or $F(D)$ in the cases (i) and (ii), respectively.

In the case (i), which is a direct problem, $S(D)$ was taken as the known input data, whereas $F(D)$ is computed from (15.1) through $F(D)=2 S(D)-S^{n}(D)$. Here, as in Refs. [314,315], we employ the single-hit-single-target sampling for the input single cell surviving fraction according to the purely exponential inactivation $S(D)=$ $\exp (-\alpha D)$. These latter cell survivals give a straight line in the semi-logarithmic plot, with $S(D)$ versus $D$ (the bottom curve in Fig. 1a). On the other hand, for e.g. $n=n_{\max }=2$, the output cell colony survival $F(D)$ is a clearly shouldered curve (the top curve in Fig. 1a). Therein, the shoulder in $F(D)$, which appears at lower doses, is most pronounced for $n=n_{\max }=2$, whereas it is flattened and stretched for $n=1.5$ (the middle curve in Fig. 1a). Of course, for $n=n_{\min }=1$, the output $F(D)$ and the input $S(D)$ coincide, $F(D)=\left\{2 S(D)-S^{n}(D)\right\}_{n=1}=S(D)$. A shoulder in a cell surviving curve is usually attributed to a repair mechanism. The results in Fig. 1a for the case (i) indicate that the inclusion of DNA replications can also produce shouldered cell surviving curves.

Conversely, in the case (ii), which is an inverse or reconstruction problem, the input data are the colony cell surviving fractions $F(D)$. Here, the task is to extract or retrieve the output single cell surviving fraction $S(D)$ from $F(D)$. These reconstructed $S(D)$ data, as the roots of the trinomial equation (15.1), have been computed from the 


\section{Cell Survival for Synchronous Populations: Corrections for Genome Multiplicity}

Direct Problem: Input Exponential Single Cell Survival Corrected for DNA Replication

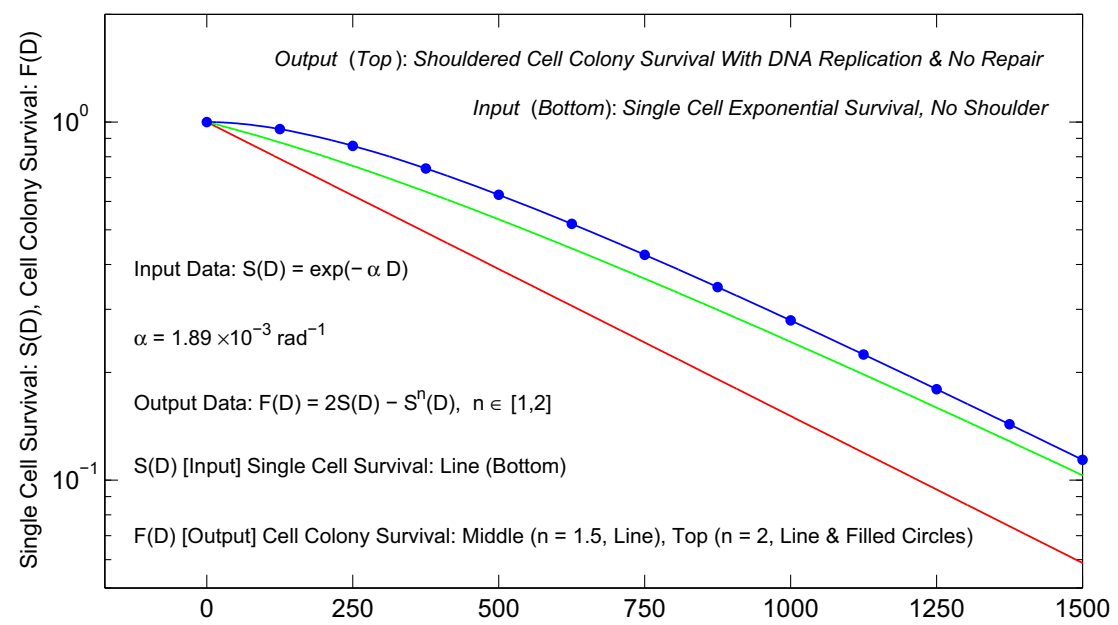

(a) Dose, $\mathrm{D}(\mathrm{rad})$

Trinomial Roots on (b) as the Fox-Wright Function for Retrieval of the Output Single Cell Survival S(D)

From the Input Cell Colony Survival F(D) Containing DNA Replication (Genome Multiplicity) \& Repair

Inverse Problem: Reconstructed Single Cell Survival from Cell Colony Survival

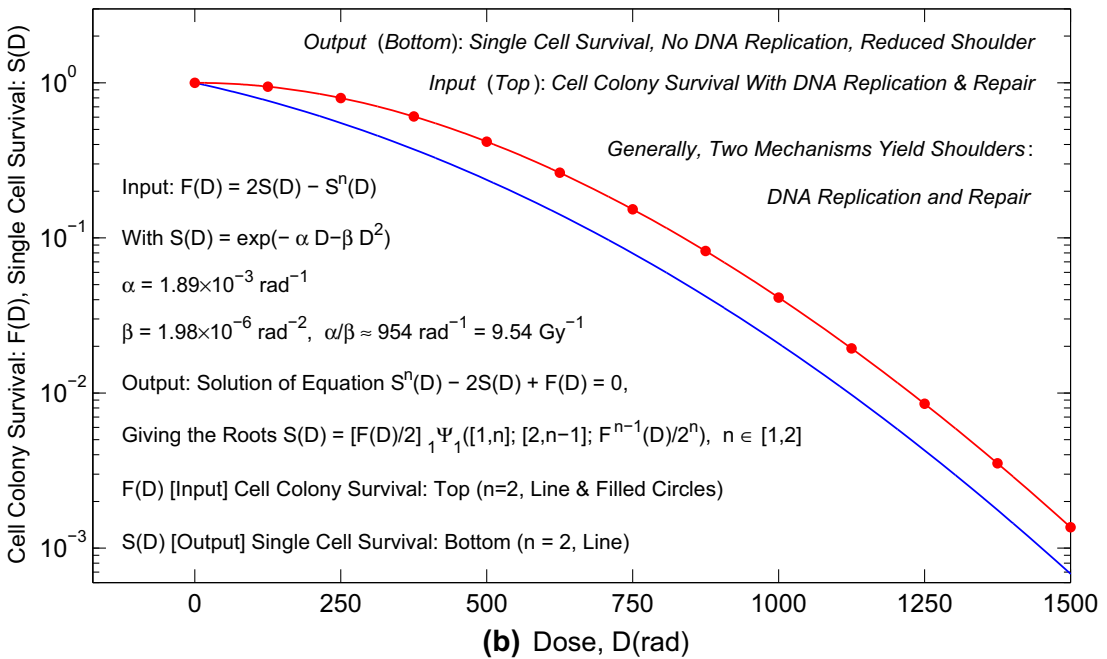

Fig. 1 Synthesized data on the corrections for genome multiplicity (DNA replications) [314,315] in survival of irradiated synchronized cell populations similar to the corresponding measured quantities from Ref. [318]. This correction, which is illustrated here on two panels, relates the single cell survival $S(D)$ to colony cell survival $F(D)$ as a function of instanteneous dose $D$. On (a), using the input $S(D)$, the output $F(D)$ is computed (a direct problem). On (b), employing the input $F(D)$, the output $S(D)$ is reconstructed as the trinomial roots (an inverse problem). The top panel shows that DNA replication can generate the shoulder with no recourse to cell repair at all. On the bottom panel, a shoulder in $S(D)$ is significantly reduced after eliminating the DNA replication component from $F(D)$ which includes both the genome multiplicity and damage repair; see the text for more details (color online) 
solution (15.3). In the case (ii), the most interesting choice corresponds to $F(D)$ which assumes that no part of DNA has been replicated. With such an input, the output $S(D)$ from (15.1) takes into account DNA replications for $n>1$. The input cell colony survival $F(D)=2 S(D)-S^{n}(D)$ is sampled with the linear-quadratic (LQ) single cell survival $S(D)=\exp \left(-\alpha D-\beta D^{2}\right)$. The ensuing data $F(D)$ for $n=2$ are shown by the top curve in Fig. 1b. We see that a departure from the straight line, which stems from the term $\exp (-\alpha D)$, appears as a prominent shoulder built from two components or mechanisms. One component is cell repair which is described in the LQ model by the Gaussian $\exp \left(-\beta D^{2}\right)$. The other component is DNA replication (or genome multiplicity). Next, starting from the sampled input data $F(D)$ for a fixed $n$, we reconstruct the output data $S(D)$. As stated, this is done by using (15.3) to compute the roots $S(D)=[F(D) / 2]_{1} \Psi_{1}\left([1, n] ;[2, n-1] ; F^{n-1}(D) / 2^{n}\right)$ of the trinomial equation $S^{n}(D)-2 S(D)+F(D)=0$ from (15.1). The resulting single cell survival data $S(D)$ for $n=2$ are displayed by the bottom curve in Fig. 1b. This latter curve for $S(D)$ has a reduced shoulder relative to the top curve for $F(D)$. The reason is that $S(D)$ has no contribution from the component due to DNA replications.

Similarly to the case (ii), in measurements with synchronized cell populations, the colony surviving fractions $F(D)$, as the input data to (15.1), contain the contributions from DNA replications and repair. In the output, the single cell surviving fraction $S(D)$ from (15.3) is void of DNA replications. Here, the trinomial equation (15.1) acts as if it were a kind of a "deconvolution" in the sense of removing the unwanted information. The unwanted information is DNA replication which is present in experimental data $F(D)$, but absent from radiobiological models. The desired information $S(D)$, with no replication in any part of DNA, being hidden in $F(D)$, is now unfolded by rooting the trinomial equation (15.1) whose roots are given by (15.3). The ensuing single cell surviving fractions $S(D)$, as the experimental data with no contribution from genome multiplicity, can be used to make the appropriate comparisons with the conventional radiobiological models that, from the onset, ignore DNA replications. In a separate publication, we shall thoroughly investigate this type of radiobiologically important applications, using the measured cell colony surviving fractions from e.g. Refs. [318324].

\section{Discussion and conclusions}

The well-known theorem by Abel proves that no algebraic solution for the roots of a general $n$th degree polynomial exists for $n>4$. Even in the important case of the simpler, $n$th degree trinomials, it is not possible to obtain the algebraic roots. An algebraic solution is the exact formula due to a finite number of steps. Of course, numerical computations can give highly accurate values of the roots e.g. by diagonalizing the equivalent Hessenberg or companion matrix which, due to its extreme sparseness, can be of a very high dimension [332-334]. Nevertheless, it is of interest to find out whether the exact zeros of the $n$th degree polynomials can be obtained analytically through e.g. an infinite number of steps, as originally suggested by Girard [335]. Such solutions are said to be non-algebraic and they can occasionally be expressed by certain special functions e.g. transcendental functions, and the like. They can be viewed as certain 
series or products that involve infinitely many steps. For example, following Girard's idea [335], it was Lambert [1] who found a series solution of the $n$th degree trinomial equation $x^{n}-x+q=0$, where $q$ is the free, constant term. Subsequently, Euler [3] symmetrized the latter equation as $x^{\alpha}-x^{\beta}=(\alpha-\beta) v x^{\alpha+\beta}$, where $\{\alpha, \beta, v\}$ are some fixed constants (none of which is necessarily an integer). He solved this equation for the roots $x$ giving a formula as a series (the Euler series). Later, several proofs of Euler's formula using various methods have been published by a number of authors, including Refs. [11,20,33-36,45,47,50].

The next step regarding the trinomial roots based upon the Euler formula would be to carry out an explicit summation of the Euler's series. The reason for having such an explicit summation (preferably in a form of one of the known special functions), is in the possibility of exploiting the established features of the identified special function. For example, of particular importance are the asymptotic behaviors of the known special functions at both small and large values of its independent variable. These asymptotes are very useful for analyzing the critical behaviors of the studied system at the two extreme conditions or situations.

We have currently proceeded towards the goal of summing up the Euler series for the root $x$ of the general trinomial equation $x-y x^{\alpha}-1=0$ (where $\alpha$ is any real or complex number) through the following steps:

- First, we carry out the proof of the Euler's formula by deriving the general expansion coefficient of the Euler series in terms of the complete multi-variate Bell polynomial $B_{n}$ (also called the exponential polynomial).

- Second, the multi-variate Bell polynomial is reduced to a much simpler univariate polynomial in terms of either the Pochhammer symbol or binomial coefficients.

- Third, the Pochhammer simplification enables the identification of the transformed series as a special function called the confluent Fox-Wright function ${ }_{1} \Psi_{1}$.

- Fourth, another confluent Fox-Wright function ${ }_{1} \Psi_{1}$ is also found for an arbitrary power (any real or complex constant) of the derived trinomial roots.

- Fifth, the logarithm of the trinomial root is expressed through a single series with the expansion coefficients in the form of either the Pochhammer symbols or the binomial coefficients.

Being an extension of the more familiar Kummer confluent hypergeometric function ${ }_{1} F_{1}$, the function ${ }_{1} \Psi_{1}$ makes the present series solutions for trinomial roots very practical, both theoretically and computationally. In theoretical developments, we can explore the known properties of the ${ }_{1} \Psi_{1}$ function (asymptotes, integral representations, etc.). Also in computations, we can use the formulae for analytical continuations into the regions beyond the original convergence radius of the ${ }_{1} \Psi_{1}$ function. This is achieved by expressing ${ }_{1} \Psi_{1}$ in variable $x$ as a linear combination of two other ${ }_{1} \Psi_{1}$ functions in another variable related to $x$. The other variable can be $1 / x$ or $x /(1-x)$ or $1-x$, etc., similarly to the existing analytical continuation formulae of the Gauss hypergeometric function ${ }_{2} F_{1}$. Even without these special transformations, analytical continuation beyond the original convergence region can also be achieved by the continued fraction representation of the ${ }_{1} \Psi_{1}$ function (in the same way as has been done for the ${ }_{2} F_{1}$ function). This is the case because a continued fraction can be expressed as a ratio of two polynomials, i.e. the Padé approximant, which is 
intrinsically an extrapolator (an analytical continuator). It should be emphasized that unlike the ${ }_{1} F_{1}$ function, which converges everywhere, the ${ }_{1} \Psi_{1}$ function converges only within its finite convergence radius. We found that the series representation of the trinomial roots and their logarithms have the same convergence radius.

An illustration is given using synthesized data for survival of irradiated cells. The simulations are reminiscent of the corresponding measured colony surviving fractions for Chinese hamster synchronized cell populations exposed to $250 \mathrm{kVp} \mathrm{X}$-rays (see the survival curve in e.g. Fig. 9 from Ref. [318] for 10.4h after incubation). Shown in the present Fig. 1 are the single cell surviving fractions $S(D)$ and the cell colony surviving fractions $F(D)$ as a function of radiation instantaneous dose $D$. Panels (a) and (b) on Fig. 1 are on the direct and inverse problems, respectively. Both panels in this figure deal with a relationship between $S(D)$ and $F(D)$. This is a trinomial relationship, $S^{n}(D)-2 S(D)+F(D)=0(1 \leq n \leq 2)$, from correcting $S(D)$ for the missing genome multiplicity $n$ (DNA replications) [314,315]. In Fig. 1a, the input and output data are $S(D)$ and $F(D)$, respectively. Conversely, in Fig. 1b, the input and output data are $F(D)$ and $S(D)$, respectively. The input bottom curve in Fig. 1a is a purely exponential survival $S(D)$ as a straight line on a semi-logarithmic scale. When this $S(D)$ is corrected for genome multiplicity with $n=1.5$ and $n=2$, the middle and the top curves are obtained in Fig. 1a for the output data $F(D)=2 S(D)-S^{n}(D)$, respectively. Here, a clearly delineated shoulder appears in the top curve of $F(D)$ for $n=2$. In Fig. 1b, the input data $F(D)$, given by the top curve, are the linear-quadratic colony surviving fractions corrected for genome multiplicity with $n=2$. Here, a pronounced shoulder is built from two components: DNA replication and radiation damage repair. When such digitized $F(D)$ data are inserted into the trinomial equation $S^{n}(D)-2 S(D)+F(D)=0$, its two-valued roots $S(D)$ are obtained for $n=2$ (computation is carried out from the present series solution for the single cell survival $S(D)$ in terms of the Fox-Wright function ${ }_{1} \Psi_{1}$ ). The smaller of the two roots, as the physical solution, is shown by the bottom curve in Fig. 1b. Therein, because $S(D)$ is void of the contribution from DNA replications, a diminished shoulder (due to repair alone) is seen in the bottom curve for $S(D)$. This is clear by reference to the top curve in Fig. 1 b for $F(D)$ whose shoulder contains both DNA replication and cell repair. Such observations are anticipated to be a further motivation for additional explorations of surviving fractions corrected for genome multiplicity. These corrections are necessary for cell populations in all the phases of the cell reproduction cycle, except the $\mathrm{G}_{1}$-phase cell population.

Acknowledgements This work is supported by the research grants from Radiumhemmet at the Karolinska University Hospital and the City Council of Stockholm (FoUU) to which the author is grateful.

Open Access This article is distributed under the terms of the Creative Commons Attribution 4.0 International License (http://creativecommons.org/licenses/by/4.0/), which permits unrestricted use, distribution, and reproduction in any medium, provided you give appropriate credit to the original author(s) and the source, provide a link to the Creative Commons license, and indicate if changes were made. 


\section{References}

1. J.H. Lambert, Observationes varie in mathesin puram. Acta Helvetica, Physico-mathematicoanatomico-botanico-medica, Basel 3, 128-168 (1758). http://www.kuttaka.org/ JHL/L1758c.pdf

2. J.H. Lambert, Observationes analitiques. Nouveaux mémoires de l'académie royale des sciences et belle-lettres, Berlin 1, 225-244 (1770)

3. L. Euler, Deformulis exponentialibus replicatis. Reprinted in Leonhard Euleri Opera Omnia, Ser. Prima, Opera Mathematica 15, 268-297 (1927) (original publication year: 1777)

4. L. Euler, De serie Lambertina plurimisque eius insignibus proprietatibus. Acta Academiae Scientarum Imperialis Petropolitinae 2, 29-51 (1783) (original publication year: 1773); reprinted in L. Euler, Opera Omnia, Series Prima, in Commentationes Algebraicae (Teubner, Leipzig, Germany, 1921), 6, 350-369 (1921). http://math.dartmouth.edu/ euler.docs/originals/E532.pdf

5. C.F. Gauss, Résolution numérique des équations trinomes. Nouv. Ann. Math. 10, 165-174 (1851)

6. J. Cockle, Sketch of a theory of transcendental roots. Philos. Mag. 20, 145-148 (1860)

7. J. Cockle, On transcendental and algebraic solution-supplemental paper. Philos. Mag. 13, 135-139 (1862)

8. R. Harley, On the theory of the transcendental solution of algebraic equations. Q. J. Math. 5, 337-361 (1862)

9. E. McClintock, An essay on the calculus of enlargement. Am. J. Math. 2, 101-161 (1879)

10. E. McClintock, Theorems in the calculus of enlargement. Am. J. Math. 17, 69-80 (1895)

11. E. McClintock, A method for calculating simultaneously all the roots of an equation. Am. J. Math. 17, 89-110 (1895) (this and the preceding paper have been read at the 1st summer meeting of the American Mathematical Society (August 14 and 15, 1894))

12. T.S. Fiske, The summer meeting of the American Mathematical Society. Bull. Am. Math. Soc. 1-6 (October 1894) (here, pages 2-4 review the above-cited 2 McClintock's papers that, as stated, have been read at the 1st summer meeting of the American Mathematical Society (August 14 and 15, 1894) and subsequently published in Am. J. Math. (op. cit.))

13. S. Günther, Eine didaktisch wichtige auflösung trinomischer gleichungen. Z. Math. Naturwiss. Unterr. 11, 68-72 (1880)

14. S. Günther, Eine didaktisch wichtige auflösung trinomischer gleichungen. Z. Math. Naturwiss. Unterr. 11, 267-267 (1880)

15. J.J. Astrand, On en ny methode for losning af trinomiske ligninger af $n^{\text {te }}$ grad. Arch. Math. Naturvidenskab Oslo 6, 448-459 (1881)

16. W. Heymann, Über die auflösung der allgemeinen trinomischen gleichung $t^{n}+a t^{n-s}+b=0$. Z. Math. Phys. 31, 223-240 (1886)

17. W. Heymann, Theorie der trinomische gleichungen. Math. Ann. 28, 61-80 (1887)

18. P. Nekrassoff, Ueber trinomische gleichungen. Math. Ann. 29, 413-430 (1887)

19. P. Bohl, Zur theorie der trinomischen gleichungen. Math. Ann. 65, 556-566 (1908)

20. P.A. Lambert, On the solution of algebraic equations in infinite series. Proc. Am. Math. Soc. 14, 467-477 (1908)

21. Hj. Mellin, Zur theorie der trinomischen gleichungen. Ann. Acad. Sci. Fenn. Ser. A 7(7), 32-73 (1915)

22. Hj. Mellin, Ein allgemeiner satz über algebraische gleichungen. Ann. Acad. Sci. Fenn. 7(8), 1-33 (1915)

23. R. Birkeland, Résolution de l'équation algébrique trinome par des fonctions hypergéométriques supérieurs. Comptes Rendus Acad. Sci. Paris 171, 778-781 (1920)

24. R. Birkeland, Résolution de l'équation algébrique genérale par des fonctions hypergéométriques des plusieurs variables. Comptes Rendus Acad. Sci. Paris 171, 1370-1372 (1920)

25. R. Birkeland, Résolution de l'équation algébrique genérale par des fonctions hypergéométriques des plusieurs variables. Comptes Rendus Acad. Sci. Paris 172, 309-311 (1921)

26. R. Birkeland, Über die auflösung algebraischer gleichungen durch hypergeometrische funktionen. Math. Z. 26, 1047-1049 (1927)

27. G. Belardinelli, L'equazione differenziale risolvente della equazione trinomia. Rend. Circ. Mat. Palermo 46, 463-472 (1922)

28. G. Belardinelli, Risoluzione analitica delle equazioni algebriche generali. Rendic. Semin. Matem. Fis. Milano 29, 13-45 (1959)

29. J. Egerváry, On the trinomial equation. Math. Phys. Lapok 37, 36-57 (1930) 
30. A.J. Kempner, Über die separation komplexer wurzeln. Math. Ann. 85, 49-59 (1922)

31. A.J. Kempner, On the separation and computation of complex roots of algebraic equations. Univ. Colo. Stud. 16, 75-87 (1928)

32. A.J. Kempner, On the complex roots of algebraic equations. Bull. Am. Math. Soc. 41, 809-84 (1935)

33. A.J. Lewis, The solutions of algebraic equations with one unknown quantity by infinite series. Ph.D. thesis, University of Colorado (1932, unpublished)

34. A.J. Lewis, The solution of algebraic equations by infinite series. Natl. Math. Mag. 10, 80-95 (1935)

35. A. Eagle, Series for all the roots of a trinomial equation. Am. Math. Mon. 46, 422-425 (1939)

36. A. Eagle, Series for all the roots of the equation $(z-a)^{m}=k(z-b)^{n}$. Am. Math. Mon. 46, 425-428 (1939)

37. A.J. Kempner, Two reviews of the preceding 2 Eagle's papers. Math. Rev. 1(1), 1-1 (1940)

38. N.A. Hall, The solution of the trinomial equation in infinite series by the method of iteration. Natl. Math. Mag. 15, 219-229 (1941)

39. L. Hibbert, Résolution des équations algébriques de la forme $z^{n}=z-a$. Comptes Rendus Acad. Sci. Paris 206, 229-231 (1938)

40. L. Hibbert, Résolution des équations $z^{n}=z-a$. Bull. Sci. Math. 63, 21-50 (1941)

41. H. Fell, The geometry of zeros of trinomial equations. Rend. Circ. Mat. Palermo 29, 303-336 (1980)

42. G. Boese, Einschlüsse und trennung der nullstellen von exponentialtrinomen. Z. Angew. Math. Mech. 62, 547-560 (1982)

43. J.D. Nulton, K.B. Stolarsky, Zeros of certain trinomials. Comptes Rendus Math. Rep. Acad. Sci. Can. 6, 243-248 (1984)

44. K. Dilcher, J.D. Nulton, K.B. Stolarsky, The zeros of a certain family of trinomials. Glasg. Math. J. 34, 55-74 (1992)

45. I.S. Moskowitz, A.R. Miller, Simple timing channels, in Proceedings of IEEE Computer Society Symposium on Research in Security and Privacy (Oakland, CA, USA, May 16-18, 1994), pp. 56-64

46. A.R. Miller, I.S. Moskowitz, Reduction of a class of Fox-Wright $\Psi$ functions for certain rational parameters. Comput. Math. Appl. 30, 73-82 (1995)

47. D.V. Chudnovsky, G.V. Chudnovsky, Classification of hypergeometric identities for $\pi$ and other logarithms of algebraic numbers. Proc. Natl. Acad. Sci. USA 95, 2744-2749 (1998)

48. P.G. Szabó, On the roots of the trinomial equation. Cent. Eur. J. Oper. Res. 18, 97-104 (2010)

49. T. Theobald, T. de Wolff, Norms of roots of trinomials. Math. Ann. 366, 219-247 (2016)

50. F. Wang, Proof of a series solution for Euler's trinomial equation. ACM Commun. Comput. Algebra 50, 136-144 (2016)

51. V. Botta, Roots of some trinomial equations. Proc. Ser. Braz. Soc. Appl. Comput. Math. 5(1), 10024 (2017). https://doi.org/10.5540/03.2017.005.01.0024

52. M.A. Brilleslyper, L.E. Schaubroeck, Counting interior roots of trinomials. Math. Mag. 91, 142-150 (2018)

53. E.T. Bell, Exponential polynomials. Ann. Math. 35, 258-277 (1934)

54. E.M. Wright, On the coefficients of power series having exponential singularities. J. Lond. Math. Soc. 8, 71-79 (1933)

55. E.M. Wright, The asymptotic expansion of the generalized Bessel function. Proc. Lond. Math. Soc. 38, 257-270 (1935)

56. E.M. Wright, The asymptotic expansion of the generalized hypergeometric function. J. Lond. Math. Soc. 10, 287-293 (1935)

57. E.M. Wright, The generalized Bessel function of order greater than one. Q. J. Math. Oxf. 11, 36-48 (1940)

58. C. Fox, The $G$ and $H$ functions as symmetrical Fourier kernels. Trans. Am. Math. Soc. 98, 395-429 (1961)

59. C.F. Faà di Bruno, Sullo sviluppo delle funzioni. Ann. Sci. Mat. Fis. 6, 479-480 (1855)

60. C.F. Faà di Bruno, Note sur une nouvelle formule de calcul différentiel. Q. J. Pure Appl. Math. 1, 359-360 (1857)

61. W.P. Johnson, The curious history of Faà di Bruno's formula. Am. Math. Mon. 109, 217-234 (2002)

62. J. Riordan, Derivatives of composite functions. Bull. Am. Math. Soc. 52, 664-667 (1946)

63. J. Riordan, An Introduction to Combinatorial Analysis (Princeton University Press, Princeton, 1978)

64. R. Gorenflo, Y. Luchko, F. Mainardi, Analytical properties and applications of the Wright function. Fract. Calc. Appl. Anal. 2, 383-414 (1999) 
65. A.A. Kilbas, M. Saigo, J.J. Trujillo, On the generalized Wright function. Fract. Calc. Appl. Anal. 5, 437-460 (2002)

66. T. Craven, G. Csordas, The Fox-Wright functions and Laguerre multiplier sequences. J. Math. Anal. Appl. 314, 109-125 (2006)

67. F. Mainardia, G. Pagninib, The role of the Fox-Wright functions in fractional sub-diffusion of distributed order. J. Comput. Appl. Math. 207, 245-257 (2007)

68. L. Michaelis, M.L. Menten, Die kinetik der invertinwirkung. Biochem. Z. 49, 333-369 (1913) (English translation by R.S. Goody and K.A. Johnson, The kinetics of invertase action. Biochem. 50, 82648269 (2011); Supporting Information: The full text (34 pp) of the German to English translation of the original paper by Michaelis and Menten (1913, op. cit.) available at: http://pubs.acs.org)

69. G.E. Briggs, J.B.S. Haldane, A note on the kinetics of enzyme action. Biochem. J. 19, 338-339 (1925)

70. M. Merriman, The solutions of equations, in Mathematical Monographs (Willey, New York, 1906, Fourth edn. enlarged), ed. by M. Merriman, R.S. Woodward, no. 10 (First edn. 1896). http://www23. us.archive.org/stream/solutionofequati00merruoft\#page/38/mode/1up

71. G. Pólya, G. Szegő, Aufgaben und Lehrsätze der Analysis (Springer, Berlin, 1925); English translation: G. Pólya, G. Szegô, Problems and Theorems in Analysis (Springer, Berlin, 1998)

72. N.I. Muskhelishvili, Singular Integral Equations (Noordhoff, Groningen, 1953)

73. G. Belardinelli, Fonctions Hypergéometric de Plusieurs Variables et Résolution Analytiques des Équations Algébraic Genérales (Gauthier-Villars, Paris, 1960)

74. N.G. de Bruijn, Asymptotic Methods in Analysis, 2nd edn. Bibliotheca Mathematica, vol. 4 (NorthHolland Publishing Company, Amsterdam, 1961)

75. R.P. Stanley, Enumerative Combinatorics, vol. 1 (Cambridge University Press, Cambridge, 1999)

76. R.P. Stanley, Enumerative Combinatorics, vol. 2 (Cambridge University Press, Cambridge, 2001)

77. D.E. Knuth, Selected Papers on the Analysis of Algorithms (Stanford University Press, Paolo Alto, 2000)

78. D.E. Knuth, Selected Papers on Discrete Mathematics (CSLI Publications, Stanford, 2003) (CSLI: Center for the Study of Language and Information)

79. E.T. Whittaker, G.N. Watson, A Course of Modern Analysis: An Introduction to the General Theory of Infinite Processes and of Analytic Functions; with an Account of the Principal Transcendental Functions (Cambridge Mathematical Library Series, 2004; Reprinted from 1996) (First edn. Cambridge University Press, Cambridge, 1902)

80. A. Etheridge, Some Mathematical Models for Population Genetics. Lecture Notes, Springer, École d'Été de Probabilités de Saint-Flour, 39 (2009) [39th Probability Summer School held in Saint-Flour]

81. S. Yi, P.W. Nelson, A.G. Ulsoy, Time Delay Systems: Analysis and Control Using the Lambert W Function (World Scientific Press, London, 2010). Supplementary material: http://www-personal. umich.edu/ ulsoy/TDS-Supplement.htm

82. R.M. Corless, N. Fillion, A Graduate Introduction to Numerical Methods (Springer, New York, 2013)

83. P.M. Pardalos, T.M. Rassias (eds.), Contributions in Mathematics and Engineering in Honor of Constantin Carathéodory (Springer, Berlin, 2016)

84. A.E. Dubinov, I.D. Dubinova, S.K. Saikov, The Lambert $W$-function and its applications to mathematical problems of physics (The Russian Federal Nuclear Center, Sarov, Russia, 2006), pp. 1-160 (in Russian)

85. R. Roy, F.W.J. Olver, Elementary functions, in NIST Handbook of Mathematical Functions (NIST: National Institute of Standards and Technology]) ed. by F.W.J. Olver, D.W. Lozier, R.F. Boisvert, C.W. Clark (NIST \& Cambridge University Press, Cambridge, New York, 2010) (Electronic version available at http://dlmf.nist.gov), Chapter 4, Sections 4.13: p. 111, 4.45(iii): p. 132

86. H. Shinozaki, Lambert $W$ approach to stability and stabilization problems for linear time-delay systems. Ph.D. thesis, Kyoto Institute of Technology, Kyoto, Japan (2007)

87. K.M. Pietarila, Developing and automating time delay system stability analysis of dynamic systems using the matrix Lambert function $W$ (MLF) method. Ph.D. thesis, University of Missouri-Colombia, USA (2008)

88. V. Letort, Adaptation du modèle de croissance GreenLab aux plantes à architecture complexe et analyse multi-échelle des relations source-puits pour l'identification paramétrique. Ph.D. thesis, École Centrale des Arts et des Manufactures, "École Centrale Paris" (2008)

89. S. Yi, Time-delay systems: analysis and control using the Lambert $W$ function. Ph.D. thesis, University of Michigan, Michigan, USA (2009) 
90. L. Wetzel, Effect of distributed delays in systems of coupled phase oscillators. Ph.D. thesis, Max Planck Institute of Complex Systems (Dresden, Germany, 2012)

91. R.M. Corless, G.H. Gonnet, D.E.G. Hare, D.J. Jeffrey, D.E. Knuth, On the Lambert $W$ function. Adv. Comput. Math. 5, 329-359 (1996)

92. J.M. Borwein, R.M. Corless, Emerging tools for experimental mathematics. Am. Math. Mon. 106, 889-909 (1999)

93. R.M. Corless, D.J. Jeffrey, The Lambert $W$ Function, in The Princeton Companion to Applied Mathematics, Chapter III.17, ed. by N.J. Higham, M. Dennis, P. Glendinning, P. Martin, F. Santosa, J. Tanner (Princeton University Press, Princeton, 2015), pp. 151-155

94. D.V. Chudnovsky, G.V. Chudnovsky, Generalized hypergeometric functions-classification of identities and explicit rational approximations, in Algebraic Methods and q-Special Functions, ed. by J.F. Van Diejen, L. Vinet, CRM Proceedings and Lecture Notes. https://doi.org/10.1090/crmp/022/ 03 (American Mathematical Society, Providence, RI, 1999), Vol. 22, pp. 59-91

95. T.D. Lamb, E.N. Pugh Jr., Phototransduction, dark adaptation, and rhodopsin regeneration (the Proctor lecture). Inv. Opht. Vis. Sci. 47, 5138-5152 (2006)

96. A.E. Dubinov, I.D. Dubinova, S.K. Saikov, The Lambert W-function and its applications to mathematical problems of physics (The Russian Federal Nuclear Center, Sarov, Russia, 2006), pp. 1-160 (in Russian)

97. P.B. Brito, F. Fabião, A. Staubyn, Euler, Lambert, and the Lambert $W$ function today. Math. Sci. 33, 127-133 (2008)

98. M. Goličnik, On the Lambert $W$ function and its utility in biochemical kinetics. Biochem. Eng. J. 63, 116-123 (2012)

99. T.P. Dance, A brief look into the Lambert $W$ function. Appl. Math. 4, 887-892 (2013)

100. Swati Sharma, P. Shokeen, B. Saini, S. Sharma Chetna, J. Kashyap, R. Guliani, Sandeep Sharma, U.M. Khanna, A. Jain, A. Kapoor, Exact analytical solutions of the parameters of different generation real solar cells using Lambert $W$-function: a review article. Int. J. Renew. Energy 4, 155-194 (2014)

101. Dž Belkić, K. Belkić, Mechanistic radiobiological models for repair of cellular radiation damage. Adv. Quantum Chem. 70, 43-143 (2015)

102. Dž Belkić, The Euler $T$ and Lambert $W$ functions in mechanistic radiobiological models with chemical kinetics for repair of irradiated cells. J. Math. Chem. 56, 2133-2193 (2018)

103. J. Lehtonen, The Lambert $\mathrm{W}$ function in ecological and evolutionary models. Methods Ecol. Evol. 2016(7), 1110-1118 (2016)

104. C. Katsimpiri, P.E. Nastou, P.M. Pardalos, Y.C. Stamation, The ubiquitous Lambert $W$ function and its classes in sciences and engineering, in Contributions in Mathematics and Engineering in Honor of Constantin Carathéodory, ed. by P.M. Pardalos, T.M. Rassias (Springer, Berlin, 2016), pp. 323-342

105. V. Barsan, Siewert solutions of the transcendental equations, generalized Lambert functions and physical applications. Open Phys. 16, 232-242 (2018)

106. Int. Workshop, "Celebrating 20 years of the Lambert $W$ function", Western University, London, Ontario, Canada (July 25-28, 2016). http://www.apmaths.uwo.ca/ djeffrey/LambertW/LambertW. html

107. Meeting on the Lambert $W$ function and other special functions in optimization and analysis, The 7th Seminar on optimization and variational analysis (Alicante, Spain, June 1-3, 2016)

108. C.E. Siewert, Publications list. https://www.ces.math.ncsu.edu/publist.html (2017)

109. K. Briggs, W-ology, or some exactly solvable growth models. http://keithbriggs.info/W-ology.html, http://morebtexact.com/people/briggsk2/W-ology.html (1999)

110. R.N. Corless, D.J. Jeffrey, Still, more fun results on the Lambert $W$ function. Maple Document. https://www.maplesoft.com/applications/view.aspx?sid=4204\&view=html (2002)

111. C. Moler, Cleve's corner: Cleve Moler on mathematics and computing; the Lambert $W$ function. Matlab program Lambert_W.m. https://blogs.mathworks.com/cleve/2013/09/02/the-lambertW-function (2013)

112. E.W. Weisstein, Lambert $W$-function. From mathworld-a Wolfram web resource. http://mathworld. wolfram.com/LambertW-Function.html (2016)

113. J.M. Borwein, S.B. Lindstrom, The Lambert function in optimization. https://carma.newcastle.edu. au/jon/WinOpt.pdf/LambertWPresentation.pdf

114. J.M. Borwein, CARMA (Computer assisted research mathematics and its applications priority). https://carma.newcastle.edu.au 
115. F. Johansson, A FLINT (Fast library for number theory) example: Lambert $W$ function power series. https://fredrik-j.blogspot.com/2011/03/flint-example-lambert-w-function-power.html

116. F. Johansson, Arbitrary precision computations of the Lambert functions, numerical quadratures, etc. http://arblib.org/ (2017)

117. G.M. Goerg, I did it the Lambert way. www.gmge.org/i-did-it-the-lambert-way/ (2010)

118. WikipediA, https://en.wikipedia.org/wiki/Talk:Lambert_W_function

119. RDdocumentation. https:/www.rdocumentation.org/packages/emdbook/versions/1.3.10/topics/ lambertW

120. Support: Online Help (Mathematics, Special Functions), The Lambert $W$ function. https://www. maplesoft.com/support/help/maple/view.aspx?path=LambertW

121. R.N. Corless, Poster: The Lambert $W$ function. http://www.orcca.on.ca/LambertW (1996)

122. A. Braun, Poster: Lambert's $W$-function in physics \& Engineering. https://www.researchgate.net/ publication/279963060_Lambert_W-Function_in_Physics_Engineering_Swiss_Physical_Society_ 2007, https://doi.org/10.13140/RG.2.1.2574.1927, Contribution to Euler's Tercentinary 2007, Swiss Physical Society (2007)

123. F.N. Fritsch, R.E. Shafer, W.P. Crowley, Algorithm 443: solution of the transcendental equation $w \mathrm{e}^{w}=x$. Commun. ACM 16, 123-124 (1973)

124. D.A. Barry, P.J. Culligan-Hensley, S.J. Barry, Real values of the W-function. Assoc. Comput. Machin. Trans. Math. Softw. 21, 161-171 (1995)

125. D.A. Barry, S.J. Barry, P.J. Culligan-Hensley, Algorithm 743: WAPR: A FORTRAN routine for calculating real values of the $W$-function. Assoc. Comput. Machin. Trans. Math. Softw. 21, 172-181 (1995)

126. D.A. Barry, J.-Y. Parlange, L. Li, H. Prommer, C.J. Cunningham, F. Stagnitti, Analytical approximations for real values of the Lambert $W$ function. Math. Comput. Simul. 53, 95-103 (2000)

127. D.H. Bailey, Y. Hida, X.S. Li, B. Thompson, Arprec: an arbitrary precision computation package. http://crd.lbl.gov/ dhbailey/dhbpapers/arprec.pdf, http://crd.lbl.gov/dhbailey/mpdist (2002)

128. D.H. Bailey, High-precision floating-point arithmetic in scientific computation. Comput. Sci. Eng. 7, 54-61 (2005)

129. P. Getreuer, Program lambertw.m. Matlab Central File Exchange (2005)

130. P. Getreuer, D. Clamond. http://www.getreuer.info/home/lambertw

131. D. Verebič, Having fun with Lambert $W(x)$ function. arXiv:1003.1628v1 [cs.MS] (2010)

132. D. Veberič, Lambert $W$ function for applications in physics. Comput. Phys. Comm. 183, 2622-2628 (2012) (open source in C++, http://cpc.cs.qub.ac.uk/summaries/AENC_v1_0.html)

133. W. Gautschi, The Lambert $W$-functions and some of their integrals: a case study for high-precision computations. Numer. Algorithm 57, 27-34 (2011)

134. P.W. Lawrence, R.M. Corless, D.J. Jeffrey. Algorithm 917: complex double-precision evaluation of the Wright $\omega$ function. ACM Trans. Math. Softw. 38, Art. 20 (2012). http://doi.acm.org/10.1145/ 2168773.2168779

135. A.D. Horchler, Complex double-precision evaluation of the Wright omega function, a solution of $\mathrm{W}+\mathrm{LOG}(\mathrm{W})=\mathrm{Z}$. GitHub, Inc. (US): Git-Respiratory Hosting Service, Microsoft Corporation (from October 2018). https://github.com/horchler/wrightOmegaq, Matlab program wrightOmegaq.m, Version 1.0, 3-12-13 (2013)

136. T. Fukushima, Precise and fast computation of Lambert $W$-functions without transcendental function evaluations. J. Comput. Appl. Math. 244, 77-89 (2013)

137. Z.L. Krougly, D.J. Jeffrey, Implementation and application of extended precision in Matlab. Math. Meth. Appl. Comput. Proc. 11th Int. Conf. Math. Meth. Comput. Techn. Electr. Eng. (Athens, 28-30 September, 2009), pp. 103-108

138. A. Adler, Lambert $W$-function (lam $W$-package, lambertW). CRAN-Package lamW, free to download: lamW_1.3.0.tar.gz, https://cran.r-project.org/web/packages/lamW/index.html, https://CRAN. R-project.org/package=lamW, https://bitbucket.org/aadler/lamw (2017)

139. F. Johansson, Computing the Lambert $W$ function in arbitrary-precision complex interval arithmetic. HAL (Arhives-Ouvertes.fr), http://hal.inria.fr/hal-01519823 (2017), http://arblib.org/, arXiv:1705:03266v1 [cs.MS]; https://arxiv.org/pdf/1705.03266.pdf (2017)

140. F. Johansson, Arb: efficient arbitrary-precision midpoint-radius interval arithmetic. IEEE Trans. Comput. http://dx.doi.org/10.1109/TC.2017.2690633 (2017)

141. F. Johansson, L. Blagouchine, Computing Stieltjes constants using complex integrations. arXiv:804.01679v2 [math.CA] (2018) 
142. F. Johansson, Numerical integration in arbitrary-precision ball arithmetic. arXiv:1802.07942 (2018)

143. J.P. Body, Global approximation of the principal real-valued branch of the Lambert $W$ function. Appl. Math. Lett. 11, 27-31 (1998)

144. S. Winitzki, Uniform approximations for transcendental functions, in Lecture Notes in Computer Science, no. 2667, ed. by V. Kumar, M.L. Gavrilova, C.J.K. Tan, P. L' Ecuyer (Springer, Berlin, 2003), pp. 780-789

145. K. Roberts, A robust approximation to a Lambert-type function. arXiv:1504.01964v1 [math.NA] (2015)

146. R. Iacono, J.P. Boyd, New approximations to the principal real-valued branch of the Lambert $W$ function. Adv. Comput. Math. 43, 1403-1436 (2017)

147. E.M. Wright, The linear difference-differential equation with constant coefficients. Proc. R. Soc. Edinb. A 62, 387-393 (1949)

148. E.M. Wright, A non-linear difference-differential equation. J. Reine Angew. Math. 194, 66-87 (1955)

149. E.M. Wright, Solution of the equation $z \mathrm{e}^{z}=a$. Proc. R. Soc. Edinb. A 65, 193-203 (1959)

150. C.E. Siewert, E.E. Burniston, An exact analytical solution of Keplers equation. Celest. Mech. 6, 294-304 (1972)

151. E.E. Burniston, C.E. Siewert, The use of Riemann problems in solving a class of transcendental equations. Proc. Camb. Philos. Soc. 73, 111-118 (1973)

152. C.E. Siewert, An exact analytical solution of an elementary critical condition. Nucl. Sci. Eng. 51, 78-79 (1973)

153. C.E. Siewert, E.E. Burniston, On a critical condition. Nucl. Sci. Eng. 52, 150-151 (1973)

154. E.E. Burniston, C.E. Siewert, Exact analytical solution of the transcendental equation $a \sin \zeta=\zeta$. SIAM J. Appl. Math. 4, 460-465 (1973)

155. C.E. Siewert, C. Essig, An exact solution of a molecular field equation in the theory of ferromagnetism. J. Appl. Math. Phys. 24, 281-286 (1973)

156. C.E. Siewert, E.E. Burniston, Exact analytical solution of $z \mathrm{e}^{z}=a$. J. Math. Anal. Appl. 73, 626-632 (1973)

157. C.E. Siewert, A.R. Burkart, On double zeros of $x=\tanh (a x+b)$. J. Appl. Math. 24, 435-439 (1973)

158. C.E. Siewert, Solutions of the equation $z \mathrm{e}^{z}=a(z+b)$. J. Math. Anal. Appl. 46, 329-337 (1974)

159. C.E. Siewert, E.E. Burniston, An exact analytical solution for the position-time relationship for an inverse-distance-squared force. Int. J. Eng. Sci. 12, 861-863 (1974)

160. C.E. Siewert, E.E. Burniston, An exact analytical solution of $x \operatorname{coth} x=\alpha x^{2}+1$. J. Comput. Appl. Math. 2, 19-26 (1976)

161. C.E. Siewert, Explicit results for the quantum-mechanical energy states basic to a finite square-well potential. J. Math. Phys. 19, 434-435 (1978)

162. C.E. Siewert, J.S. Phelps III, On solutions of a transcendental equation basic to the theory of vibrating plates. SIAM J. Math. Anal. 10, 105-108 (1979)

163. C.E. Siewert, J.S. Phelps, Explicit solutions of $a \tan (\xi k \pi)+\tanh \xi=0$. J. Comput. Appl. Math. 5, 99-103 (1979)

164. C.E. Siewert, An exact expression for the Wien displacement constant. J. Quant. Spectr. Radiat. Transf. 26, 467-467 (1981)

165. R.M. Corless, G.H. Gonnet, D.E.G. Hare, D.J. Jeffrey, Lambert's $W$ function in Maple. Maple Tech. Newslett. 9, 12-22 (1993). http://www.orcca.on.ca/LambertW

166. D.J. Jeffrey, R.M. Corless, D.E.G. Hare, D.E. Knuth, Sur l'inversion de $y^{\alpha} \mathrm{e}^{y}$ au moyen de nombres de Stirling associés. Comptes Rendus Acad. Sci. Paris Série I 320, 1449-1452 (1995)

167. D.J. Jeffrey, D.E.G. Hare, R.M. Corless, Unwinding the branches of the Lambert function. Math. Sci. 21, 1-7 (1996)

168. R.M. Corless, D.J. Jeffrey, A sequence of series for the Lambert $W$ function, in Proceedings of International Symposium on Symbolic and Algebraic Computation [Kihei, Havaii, US], ed. by W.W. Kuechlin (ACM Press, New York, 1997), pp. 197-204. https://doi.org/10.1145/258726.258783

169. R.M. Corless, D.J. Jeffrey, On the Wright $\omega$ function. http://www.orcca.on.ca/TechReports/ TechReports/2000/TR-00-12.pdf (2000)

170. R.M. Corless, D.J. Jeffrey, On the Wright $\omega$ function, in Proceedings of International Joint Conference on Artificial Intelligence Automat. Reason. Symb. Comput. ed. by J. Calmet, B. Benhamou, O. Caprotti, L. Henocque, V. Sorge (Spring, London, 2002), pp. 76-89

171. S.R. Valluri, D.J. Jeffrey, R.M. Corless, Some applications of the Lambert $W$ function in physics. Can. J. Phys. 78, 823-831 (2000) 
172. R.M. Corless, D.J. Jeffrey, The Wright omega function, in Artificial Intelligence, Automated Reasoning, and Symbolic Computation, Joint International Conferences, AISC 2002 and Calculemus 2002, Marseille, France, July 2002 ed. by J. Calmet, B. Benhamou, O. Caprotti, L. Henocque, V. Sorge http://orcca.on.ca/TechReports/2000/TR-00-12.html (Springer, Berlin, 2002), pp. 76-89

173. J.M. Hefferman, R.M. Corless, Solving some delay differential equations with computer algebra. Math. Sci. 31, 21-34 (2006)

174. R.M. Corless, H. Ding, N.J. Higham, D.J. Jeffrey, The solution of $S \exp (S)=A$ is not always the Lambert function of $A$, in Proceedings of the International Symposium on Symbolic and Algebraic Computation (Waterloo, Ontario, Canada, 2007), pp. 116-121

175. M. Bronstein, R.M. Corless, J.H. Davenport, D.J. Jeffrey, Algebraic properties of the Lambert W function from a result of Rosenlicht and of Liouville. Integral Transf. Spec. Funct. 19, 709-712 (2008)

176. G.A. Kalugin, D.J. Jeffrey, R.M. Corless, P.B. Borwein, Stieltjes and other integral representations for functions of Lambert $W$. Integral Transf. Spec. Funct. 23, 581-593 (2011)

177. G.A. Kalugin, D.J. Jeffrey, R.M. Corless, Bernstein, Pick, Poisson and related integral expressions for Lambert $W$. Integral Transf. Spec. Funct. 23, 817-829 (2012)

178. T.C. Scott, J.F. Babb, A. Dalgarno, J.D. Morgan III, Resolution of a paradox in the calculation of exchange forces for $\mathrm{H}_{2}^{+}$. Chem. Phys. Lett. 203, 175-183 (1993)

179. T.C. Scott, J.F. Babb, A. Dalgarno, J.D. Morgan III, Calculation of exchange forces: general results and specific models. J. Chem. Phys. 99, 2841-2854 (1993)

180. T.C. Scott, M. Aubert-Frécon, J. Grotendorst, New approach for the electronic energies of the hydrogen molecular ion. Chem. Phys. 324, 323-338 (2006)

181. T.C. Scott, R. Mann, R.E. Martinez II, General relativity and quantum mechanics: towards a generalization of the Lambert $W$ function. Appl. Algor. Eng. Commun. Comput. 17, 41-47 (2006) (A longer version, with the same authors \& the title is in: Forschungszentrum Juelich Technical Report Nr. FZJ-ZAM-IB-2005-10)

182. T.C. Scott, G. Fee, J. Grotendorst, Asymptotic series of generalized Lambert $W$ functions. ACM Commun. Comput. Algorithm 47, 75-83 (2013)

183. T.C. Scott, G. Fee, W.H. Zhang, Numerics of the generalized Lambert $W$ function. ACM Commun. Comput. Algorithm 48, 42-56 (2014)

184. A. Maignan, T.C. Scott, Fleshing out the generalized Lambert $W$ function. ACM Commun. Comput. Algorithm 50, 45-60 (2016)

185. N.D. Hayes, The roots of the equation $x=(c \exp )^{n} x$ and the cycles of the substitution $\left(x \mid c \mathrm{e}^{x}\right)$. Q. J. Math. Oxf. 3, 81-90 (1952)

186. G.N. Raney, Functional composition patterns and power series reversion. Am. Math. Soc. 94, 411-451 (1960)

187. G.N. Raney, A formal solution of $\sum_{i=1}^{\infty} A_{i} \mathrm{e}^{B_{i} X}=X$. Can. J. Math. 16, 755-762 (1964)

188. N.I. Iokamidis, Application of the generalized Siewert-Burniston method to locating zeros and poles of meromorphic functions. Z. Angew. Math. Phys. 36, 733-742 (1985)

189. N.I. Iokamidis, E.G. Anastasselou, On the simultaneous determination of zeros of analytic or sectionally analytic functions. Computing 36, 239-247 (1986)

190. N.I. Ioakimidis, A note on the closed-form determination of zeros and poles of generalized analytic functions. Stud. Appl. Math. 81, 265-269 (1989)

191. D. Kalman, A generalized logarithm for exponential-linear equations. Coll. Math. J. 32, 2-14 (2001)

192. F. Chapeau-Blondeau, A. Monir, Numerical evaluation of the Lambert $W$ function and application to generation of generalized Gaussian noise with exponent 1/2. IEEE Trans. Signal Process. 50, 2160-2165 (2002)

193. D.A. Barry, L. Li, D.-S. Jeng, Comments on numerical evaluation of the Lambert $W$ function and application to generation of generalized Gaussian noise with exponent 1/2. IEEE Trans. Signal Process. 52, 1456-1458 (2004)

194. C. Moler, Stiff differential equations. Matlab News \& Notes, 12-13 (May 2003)

195. G.D. Scarpello, D. Ritelli, A new method for explicit integration of Lotka-Volterra equations. Divulg. Mat. 11, 1-17 (2003)

196. S.-D. Shih, Comment on 'A new method for explicit integration of Lotka-Volterra equations'. Divulg. Mat. 13, 99-106 (2005)

197. A.I. Kheyfits, Closed-form representations of the Lambert $W$ function. Fract. Calc. Appl. Anal. 7, 177-190 (2004) 
198. I.N. Galiadakis, On the application of the Lambert's $W$ function to infinite exponentials. Complex Var. 49, 759-780 (2004)

199. I.N. Galidakis, On solving the $p$-th complex auxiliary equation $f^{(p)}(z)=z$. Complex Var. 50, 977-997 (2005)

200. I.N. Galiadakis, On, some applications of the generalized hyper-Lambert functions. Complex Var. Elliptic Equ. 52, 1101-1119 (2007)

201. G.A. Kalugin, D.J. Jeffrey, Unimodal sequences show Lambert $W$ is Bernstein. Comptes Rendus Math. Acad. Sci. Soc. R. Can. 33, 50-56 (2011)

202. I. Mező, G. Keady, Some physical applications of generalized Lambert functions. Eur. J. Phys. 37, Art. ID 065802 (2016)

203. I. Mező, On the structure of the solution set of a generalized Euler-Lambert equation. J. Math. Anal. Appl. 455, 538-553 (2017)

204. I. Mezô, Á. Baricz, On the generalization of the Lambert $W$ function. Trans. Am. Math. Soc. 369, 7917-7934 (2017)

205. G. Keady, N. Khajohnsaksumeth, B. Wiwatanapataphee, On functions and inverses, both positive, decreasing and convex: And Stieltjes functions. Cog. Math. Stat. 5, Art. ID 1477543 (2018)

206. E.G. Anastasselou, N.I. Ioakimidis, A generalization of the Siewert-Burniston method for the determination of zeros of analytic functions. J. Math. Phys. 25, 2422-2425 (2017)

207. G.M. Goerg, Lambert $W$ random variables_a new family of generalized skewed distributions with applications of risk estimations. Ann. Appl. Stat. 5, 2197-2230 (2011)

208. D.J. Jeffrey, G.A. Kalugin, N. Murdoch, Lagrange inversion of the Lambert $W$ function, in International Symposium on Symbolic and Numeric Algorithms for Scientific Computing (Timisoara, Romania, September 21-24, 2015), pp. 42-46

209. M. Josuat-Vergès, Derivatives of the tree function. Ramanujan J. 38, 1-15 (2015)

210. L. Bertoli-Barsotti, T. Lando, A theoretical model of the relationship between the $h$-index and other simple citation indicators. Scientometrics 111, 1415-1448 (2017)

211. L. Bertoli-Barsotti, T. Lando, The $h$-index as an almost exact function of some basic statistics. Scientometrics 113, 1209-1228 (2017)

212. S. Gnanarajan, Solutions of the exponential equation $y^{x / y}=x$ or $(\ln x) / x=(\ln y) / y$ and fine structure constants. J. Appl. Math. Phys. 5, 74192 (2017)

213. S. Gnanarajan, Solutions for series of exponential equations in terms of Lambert $W$ function and fundamental constants. J. Appl. Math. Phys. 6, 725-736 (2018)

214. Y.Q. Chen, K.L. Moore, Analytical stability for delayed second-order systems with repeating poles using the Lambert $W$ function. Automatica 38, 891-895 (2002)

215. Y.Q. Chen, K.L. Moore, Analytical stability bound for a class of delayed fractional-order dynamic systems. Nonlinear Dyn. 29, 191-200 (2002)

216. Q.-C. Zhong, Robust stability analysis of simple systems controlled over communication networks. Automatica 39, 1309-1312 (2003)

217. C. Hwang, Y.-C. Cheng, A note of the use of the Lambert $W$ function in the stability analysis of the time-delay systems. Automatica 41, 1979-1985 (2005)

218. T. Kalmár-Nagy, A novel method for efficient numerical stability analysis of delay-differential equations, American Control Conference (AACC: June 8-10, 2005, Portland, Oregon, USA), ThB18.2, pp. 2823-2826

219. H. Shinozaki, T. Mori, Robust stability analysis of linear time-delay systems by Lambert $W$ function: some extreme point results. Automatica 42, 1791-1799 (2006)

220. W. Deng, J. Lu, C. Li, Stability of N-dimensional linear systems with multiple delays and application to synchronization. J. Syst. Sci. Complex. 19, 149-156 (2006)

221. Z.H. Wang, H.Y. Hu, Calculation of the rightmost characteristic root of retarded time-delay systems via Lambert $W$ function. J. Sound Vib. 318, 757-767 (2008)

222. S. Yi, P.W. Nelson, A.G. Ulsoy, Delay differential equations via the matrix Lambert $W$ function and bifurcation analysis: applications to machine tool clutter. Math. Biosci. Eng. 4, 355-368 (2007)

223. S. Yi, P.W. Nelson, A.G. Ulsoy, The use of the Lambert method with delays and with structure having the form of the star. Int. J. Inf. Educ. Technol. 2, 360-363 (2012)

224. B. Cogan, A.M. Paor, Analytical root locus and Lambert $W$ function in a control of a process with time delay. J. Electr. Eng. 62, 327-334 (2011) 
225. J. Mech, D. Bandopadhya, Applications of the Lambert $W$-function for solving time-delayed response of smart material actuator under alternating electric potential. Proc. Inst. Mech. Eng. C: J. Mech. Eng. Sci. 230, 2135-2144 (2015)

226. J.W. Müller, Deux nouvelles expressions concernant un temps mort cumulatif. BIPM Working Party Note 217, 5 (1980). www.bipm.org/en/publications/wpn.html (BIPM: Bureau Internationale des Poids et Mesures)

227. B.A. Magradze, An analytic approach to perturbative QCD. Int. J. Mod. Phys. A 15, 2715-2733 (2000)

228. J.-M. Caillol, Some applications of the Lambert $W$ function to classical statistical mechanics. J. Phys. A 36, 10431-10442 (2003)

229. A.E. Dubinov, I.N. Galiadkis, Explicit solution of the Kepler equation. Phys. Part. Nucl. Lett. 4, 213-216 (2007)

230. C. Huang, Z. Cai, C. Ye, H. Xu, Explicit solution of Raman fiber laser using Lambert $W$ function. Opt. Exp. 15, 4671-4676 (2007)

231. S.R. Valluri, M. Gil, D.J. Jeffrey, S. Basu, The Lambert $W$ function in quantum statistics. J. Math. Phys. 50, Art. ID 102103 (2009)

232. S.R. Valluri, P. Wiegert, J. Drozd, M. Da Silva, A study of the orbits of the logarithmic potential for galaxies. Month. Not. R. Astron. Soc. 427, 2392-2400 (2012)

233. P.A. Karkantzakos, Time of flight and the range of the motion of a projectile in a constant gravitational field under the influence of a retarding force proportional to the velocity. J. Eng. Sci. Technol. Rev. 2, 76-81 (2009)

234. D. Gamliel, Using the Lambert function in an exchange NMR process with a time delay. Electronics J. Qualit. Theory Diff. Equat. (Proc. 9th Coll. Qualit. Theory Diff. Equat.) no. 7, 1-12 (2012)

235. A. Houari, Additional applications of Lambert $W$ function in physics. Eur. J. Phys. 34, 695-702 (2013)

236. A.M. Ishkhanyan, A singular Lambert- $W$ Schrödinger potential exactly solvable in terms of the confluent hypergeometric function. Mod. Phys. Lett. 31, Art. ID 1650177 (2016)

237. D.A. Morales, Exact expressions for the range and the optimal angle of a projectile with linear drag. Can. J. Phys. 83, 67-83 (2005)

238. D.A. Morales, A generalization on projectile motion with linear resistance. Can. J. Phys. 89, 12331250 (2011)

239. D.A. Morales, Relationships between the optimum parameters of four projectile motions. Acta Mech. 227, 1593-1607 (2016)

240. E.W. Packel, D.S. Yuen, Projectile motion with resistance and the Lambert function. Coll. Math. J. 35, 337-350 (2004)

241. R.D.H. Warburton, J. Wang, Analysis of asymptotic projectile motion with air resistance using the Lambert $W$ function. Am. J. Phys. 72, 1404-1407 (2004)

242. R.D.H. Warburton, J. Wang, J. Burgdörfer, Analytical approximations of projectile motion with quadratic air resistance. J. Serv. Sci. Manag. 3, 98-105 (2010)

243. S.M. Stewart, Linear resisted projectile motion and the Lambert $W$ function. Am. J. Phys. 73, 199-199 (2005)

244. S.M. Stewart, An analytical approach to projectile motion in a linear resisting medium. Int. J. Math. Educ. Sci. Technol. 37, 411-431 (2006)

245. S.M. Stewart, On certain inequalities involving the Lambert $W$ function. Inequal. Pure. Appl. Math. 10(4), Art. ID 96 (2009)

246. S.M. Stewart, Some remarks on the time of flight and range of a projectile in a linear resisting medium. J. Eng. Sci. Technol. Rev. 4, 32-34 (2011)

247. H. Hernández-Saldaña, On the locus formed by the maximum heights of projectile motion with air resistance. Eur. J. Phys. 31, 1319-1329 (2010)

248. S.M. Stewart, Comment on 'On the locus formed by the maximum heights of projectile motion with air resistance'. Eur. J. Phys. 32, L7-L10 (2011)

249. S.M. Stewart, On the trajectories of projectiles depicted in early ballistic woodcuts. Eur. J. Phys. 33, 149-166 (2012)

250. H. Hu, Y.P. Zhao, Y.J. Guo, M.J. Zheng, Analysis of linear resisted projectile motion using the Lambert $W$ function. Acta Mech. 223, 441-447 (2012)

251. R.C. Bernardo, J.P.H. Esguerra, J.D. Vallejos, J.J. Canda, Wind-influenced projectile motion. Eur. J. Phys. 36, Art. ID 025016 (2015) 
252. S.R. Cranmer, New views of the solar wind with the Lambert $W$ function. Am. J. Phys. 72, 1397-1403 (2004). http://cfa-www.harvard.edu/scranmer/News2004/

253. A. Jain, A. Kapoor, Exact analytical solutions of the parameters of real solar cells using Lambert $W$-function. Sol. Energy Mater. Sol. Cells 81, 269-277 (2004)

254. A. Jain, A. Kapoor, A new approach to study organic solar cell using Lambert $W$-function. Sol. Energy Mater. Sol. Cells 86, 197-205 (2005)

255. F. Ghani, M. Duke, Numerical determination of parasitic resistances of a solar cell using the Lambert $W$-function. Sol. Energy 85, 2386-2394 (2011)

256. J. Cubas, S. Pindado, C. de Manuel, Explicit expressions for solar panel equivalent circuit parameters based on analytical formulation and the Lambert $W$-function. Energies 7, 4098-4115 (2014)

257. P. Upadhyay, S. Pulipaka, M. Sharma, Parametric extraction of solar photovoltaic system using Lambert $W$ function for different environment condition. IEEE Conf. TENCON (Singapore, November 22-25, 2016) 32, 1884-1888 (2016)

258. J. Ge, M. Luo, W. Pan, Na Li, W. Peng, Parameters extraction for perovskite solar cells based on Lambert $W$-function. MATEC Web Conf. 59, Art. ID 03003 (2016)

259. K. Roberts, S.R. Valluri, Solar cells and the Lambert $W$ function. https://www.researchgate.net/ publication/305991463 (2016), Int. Workshop, "Celebrating 20 years of the Lambert $W$ function", Western University, London, Ontario, Canada (July 25-28, 2016). www.apmaths.uwo.ca/ djeffrey/ LambertW/LambertW.html

260. S. Schnell, C. Mendoza, Closed form solution for time-dependent enzyme kinetics. J. Theor. Biol. 187, 202-212 (1997)

261. S. Schnell, C. Mendoza, Time-dependent closed form solutions for fully competitive enzyme reactions. Bull. Math. Biol. 62, 321-336 (2000)

262. S. Schnell, P.K. Maini, Enzyme kinetics at high enzyme concentrations. Bull. Math. Biol. 62, 483-499 (2000)

263. C.T. Goudar, J.R. Sonnad, R.G. Duggleby, Parameter estimation using a direct solution of the integrated Michaelis-Menten equation. Biochim. Biophys. Acta 1429, 377-383 (1999)

264. C.T. Goudar, S.K. Harris, M.J. McInerney, J.M. Suflita, Progress curve analysis for enzyme and microbial kinetic reactions using explicit solutions based on the Lambert $W$ function. J. Microbiol. Methods 59, 317-326 (2004)

265. M.V. Putz, A.-M. Lacrămă, V. Ostafe, Full analytic progress curves of enzymatic reactions in vitro. Int. J. Mol. Sci. 2006, 469-484 (2006)

266. M.V. Putz, A.-M. Lacrămă, V. Ostafe, Introducing logistic enzyme kinetics. J. Optoelectron. Adv. Mater. 9, 2910-2916 (2007)

267. M. Helfgott, E. Seier, Some mathematical and statistical aspects of enzyme kinetics. J. Online Math. Appl. 7, 1611 (2007). http://www.maa.org/joma/Volume7/HelfEnzyme.pdf

268. M.N. Berberan-Santos, A general treatment of Henri-Michaelis-Menten enzyme kinetics: exact series solution and approximate analytical solutions. MATCH Commun. Math. Comput. Chem. 63, 283-318 (2010)

269. C. Baleizão, M.N. Berberan-Santos, Enzyme kinetics with a twist. J. Math. Chem. 49, 1949-1960 (2011)

270. M. Goličnik, Explicit reformulations of time-dependent solution for a Michaelis-Menten enzyme reaction model. Anal. Biochem. 406, 94-96 (2010)

271. M. Goličnik, Explicit analytic approximations for time-dependent solutions of the integrated Michaelis-Menten equation. Anal. Biochem. 411, 303-305 (2011)

272. M. Goličnik, Exact and approximate solutions of the decades old Michaelis-Menten equation: progress curve analysis through integrated rate equations. Biochem. Mol. Biol. Educ. 39, 117-125 (2011)

273. M. Goličnik, Evaluation of enzyme kinetic parameters using explicit analytical approximations to the solution of the Michaelis-Menten equation. Biochem. Eng. J. 53, 234-238 (2011)

274. F. Exnowitz, B. Meyer, T. Hackl, NMR for direct determination of $K_{\mathrm{m}}$ and $V_{\max }$ of enzyme reactions based on the Lambert $W$ function-analysis of progress curves. Biochim. Biophys. Acta 1824, 443-449 (2012)

275. L. Bayón, J.A. Otero, P.M. Suárez, C. Tasis, Solving linear unbranched pathways with MichaelisMenten kinetics using the Lambert $W$-function. J. Math. Chem. 54, 1351-1369 (2016)

276. F. Bäuerle, A. Zotter, G. Schreiber, Direct determination of enzyme kinetic parameters from single reactions using a new progress curve analysis tool. Protein Eng. Des. Sel. 30, 151-158 (2017) 
277. D.P. Francis, K. Willson, L.C. Davies, A.J.S. Coats, M. Piepoli, Quantitative general theory for periodic breathing in chronic heart failure and its clinical implications. Circulation 102, 2214-2221 (2000)

278. K. Sigmudsson, G. Másson, R. Rice, M. Beauchemin, B. Öbrink, Determination of active concentrations and associations and dissociation rate constants of interacting biomolecules: an analytical solution to the theory for kinetic and mass transport limitation and biosensor technology and its experimental verifications. Biochemistry 41, 8263-8276 (2002)

279. O.A.R. Mahroo, T.D. Lamb, Recovery of the human photopic electroretinogram after bleaching exposures: estimation of pigment regeneration kinetics. J. Physiol. 554, 417-437 (2004)

280. T.D. Lamb, E.N. Pugh Jr., Dark adaptation and the retinoid cycle of vision. Prog. Retin. Eye Res. 23, 307-380 (2004)

281. D. Van De Ville, T. Blu, M. Unser, Wavelet-based fMRI statistical analysis approach and spatial interpolation: a unifying approach, in The 2nd IEEE International Symposium on Biomedical Imaging: Nano to Macro (IEEE Cat No. 04EX821), pp. 1167-1170 (2004)

282. K. Smallbonea, D.J. Gavaghanb, R.A. Gatenbyc, P.K. Mainia, The role of acidity in solid tumour growth and invasion. J. Theor. Biol. 235, 476-484 (2005)

283. J. Li, Y. Kuang, C. Mason, Modeling the glucose-insulin regulatory system and ultradian insulin secretory oscillations with two time delays. J. Theor. Biol. 242, 722-736 (2006)

284. R.C. Sotero, Y. Iturria-Medina, From blood oxygenation level dependent (BOLD) signals to brain temperature maps. Bull. Math. Biol. 73, 2731-2747 (2011)

285. X.-S. Wang, J. Wu, Y. Yang, Richards model revisited: Validation by and application to infection dynamics. J. Theor. Biol. 313, 12-19 (2012)

286. Dž Belkić, Survival of radiation-damaged cells via mechanism of repair by pool molecules: the Lambert function as the exact analytical solution of coupled kinetic equations. J. Math. Chem. 52, 1201-1252 (2014)

287. Dž Belkić, Repair of irradiated cells by Michaelis-Menten enzyme catalysis: the Lambert function for integrated rate equations in description of surviving fractions. J. Math. Chem. 52, 1253-1291 (2014)

288. R. Nagarajan, K. Krishnau, C. Monica, Stability analysis of a glucose-insulin dynamic system using matrix Lambert $W$ function. Br. J. Math. Comput. Sci. 8, 112-120 (2015)

289. J.R. Sonnad, C.T. Goudar, Explicit reformulation of the Colebrook-White equation for turbulent flow friction factor calculation. Ind. Eng. Chem. Res. 46, 2593-2600 (2007)

290. D. Clamond, Efficient resolution of the Colebrook equation. Ind. Eng. Chem. Res. 48, 3665-3671 (2009)

291. D. Brkić, $W$ solution to the CW equation for flow friction. Appl. Math. Lett. 24, 1379-1383 (2011)

292. P. Praks, D. Brkić, Advanced iterative procedures for solving the implicit Colebrook equation for fluid flow friction. Adv. Civ. Eng. 2018, Art. ID 5451034 (2018)

293. S. Li, Y. Liu, Analytical and explicit solutions to implicit wave friction-factor equations based on the Lambert $W$ function. J. Coast. Res. (2018). https://doi.org/10.2112/JCOASTRES-D-17-00181.1

294. D. Polovinka, B. Nevzlyn, A. Syrtsov, Measuring device moisture content of transformer oil. TEKA 13, 191-200 (2013) (TEKA: Commission of Motorization and Energetics in Agriculture; Int. J. of Motorization, Vehicle Operation, Energy Efficiency and Mechanical Engineering)

295. S.M. Disney, R.D.H. Warburton, On the Lambert $W$ function: economic order quality applications and pedagogical considerations. Int. J. Product. Econ. 140, 756-764 (2012)

296. J. Gómez-Gardeñes, L. Lotero, S.N. Taraskin, F.J. Pérez-Reche, Explosive contagion in networks. Sci. Rep. 6, Art. ID 19767 (2016). https://doi.org/10.1145/258726.258783

297. B. Das, Obtaining Wien's displacement law from Planck's law of radiation. Phys. Teach. 40, 148-149 (2002). https://doi.org/10.1119/1.1466547

298. F.Q. Gouvea, Time for a new elementary function. Editorial in: FOCUS 20, 2 (2002) (FOCUS: Newsletter of Mathematics Association of America)

299. S.M. Stewart, A new elementary function for our curricula? Aust. Senior Math. J. 19, 8-26 (2002)

300. S.M. Stewart, A little introductory and intermediate physics with the Lambert $W$ function. Proc. 16th Aust. Inst. Phys. 5, 194-197 (2005)

301. S.M. Stewart, Wien peaks and the Lambert $W$ function. Revista Brasileira de Ensino de Fízica 33, Art. ID 3308 (2011)

302. S.M. Stewart, Spectral peaks and Wien's displacement law. J. Thermophys. Heat Transf. 26, 689-691 (2012) 
303. E.W. Packel, The Lambert W function and undergraduate mathematics, in Essays in Mathematics and Statistics, ed. by V. Akis, ATINER, Athens, Greece, 2009, pp. 147-154

304. B.W. Williams, The utility of the Lambert function $W[\operatorname{aexp}(a-b t)]$ in chemical kinetics. J. Chem. Educ. 87, 647-651 (2010)

305. B.W. Williams, A specific mathematical form for Wien's displacement law as $v_{\max } T=$ constant. J. Chem. Educ. 91, 623-623 (2014)

306. S.G. Kazakova, E.S. Pisanova, Some applications of the Lambert $W$-function to theoretical physics education. CP1203, Proc. 7th Int. Conf. Balkan Phys. Union, ed. by A. Angelopoulos, T. Fildisis, Amer. Inst. Phys. pp. 1354-1359 (2010), https://www.researchgate.net/publication/252985023_ Some_applications_of_the_-Lambert_W-function_to_Theoretical_Physics_Education

307. A. Vial, Fall with linear drag and Wien's displacement law: approximate solution and Lambert function. Eur. J. Phys. 33, 751-755 (2012)

308. D.W. Ball, Wien's displacement law as a function of frequency. J. Chem. Educ. 90, 1250-1252 (2013)

309. R. Das, Wavelength- and frequency-dependent formulations of Wien's displacement law. J. Chem. Educ. 92, 1130-1134 (2015)

310. R.M. Digilov, Gravity discharge vessel revisited: an explicit Lambert $W$ function solution. Am. J. Phys. 85, 510-514 (2017)

311. K. Roberts, S.R. Valluri, Tutorial: The quantum finite square well and the Lambert $W$ function. Can. J. Phys. 95, 105-110 (2017)

312. D. Brkić, Solution of the implicit Colebrook equation for flow friction using excel. Spreadsheets Educ. 10(2), Art, 2 (2017)

313. M. Ito, Lambert $\mathrm{W}$ function and hanging chain revisited. Researchgate, https://www. researchgate.net/publication/327763764 [Lambert_W_function_and_hanging_chain_revisited.pdf], arXiv:1809.07047v1 [physics.class-ph] (2018)

314. C.J. Gillespie, J.D. Chapman, A.P. Reuvers, D.L. Dugle, The inactivation of Chinese hamster cells by X rays: synchronized and exponential cell populations. Radiat. Res. 64, 353-364 (1975)

315. C.J. Gillespie, J.D. Chapman, A.P. Reuvers, D.L. Dugle, Survival of X-irradiated hamster cells: analysis of the Chadwick-Leenhouts model, in Cellular Survival after Low Doses of Irradiation, The 6th L.H. Gray Conf. Proc. (Wiley, The Institute of Physics Publishing, Bristol, 1975), pp. 25-63

316. A. Brahme, Biologically optimized 3-dimensional in vivo predictive assay-based radiation therapy using positron emission tomography-computerized tomography imaging. Acta Oncol. 42, 123-136 (2003)

317. L. Comtet, Advanced Combinatorics (D. Reidel Publishing Company, Dordrecht, 1974)

318. W.K. Sinclair, R.A. Morton, X-ray and ultraviolet sensitivity of syncronized Chinese hanster cells at various stages of the cell cycle. Biophys. J. 5, 1-25 (1965)

319. W.K. Sinclair, R.A. Morton, X-ray sensitivity during the cell generation cycle of cultured Chinese hamster cells. Radiat. Res. 29, 450-474 (1966)

320. W.K. Sinclair, The shape of radiation survival curves of mammalian cells cultered in vitro, in Biophysical Aspects of Radiation Quality (International Atomic Energy Agency, IAEA, Vienna, 1966), Technical Report Series 58, pp. 21-43

321. W.K. Sinclair, Cyclic X-ray responses in mammalian cells in vitro. Radiat. Res. 33, 620-643 (1968)

322. J. Kruuv, W.K. Sinclair, X-Ray sensitivity of synchronized Chinese hamster cells irradiated during hypoxia. Radiat. Res. 36, 45-54 (1968)

323. W.K. Sinclair, Protection by cysteamine against lethal X-ray damage during the cell cycle of Chinese hamster cells. Radiat. Res. 39, 135-154 (1969)

324. W.K. Sinclair, Dependence of radiosensitivity upon cell age, in Conference on Time and Dose Relationships in Radiation Biology as Applied to Radiotherapy (held at Carmel, California, September 15-18, 1969), Brookhaven National Laboratory Technical Report, \# BNL-50203 (C-57), pp. 97-116

325. S. Biade, C.C. Stobbe, J.D. Chapman, The intrinsic radiosensitivity of some human tumor cells throughout their cell cycles. Radiat. Res. 147, 416-421 (1997)

326. J.D. Chapman, C.J. Gillespie, Radiation-induced events and their time scale in mammalian cells. Adv. Radiat. Biol. 9, 143-198 (1981)

327. J.D. Chapman, C.J. Gillespie, The power of radiation biophysics-let's use it. Int. J. Radiat. Oncol. Biol. Phys. 84, 309-311 (2012)

328. J.D. Chapman, A.E. Nahum, Radiotherapy Treatment Planning: Linear-Quadratic Radiobiology (Taylor \& Francis, London, 2016), Ch 3, Intrinsic radiosensitivity of proliferating and quiescent cells, pp. 19-30 
329. K.H. Chadwick, H.P. Leenhouts, The effect of an asynchronous population of cells on the initial slope of dose-effect curve, in Cellular Survival after Low Doses of Irradiation, The 6th L.H.Gray Conf. Proc. (Wiley, The Institute of Physics Publishing, Bristol, 1975), pp. 57-63

330. K.H. Chadwick, H.P. Leenhouts, The Molecular Theory of Radiation Biology (Springer, Heidelberg, 1981), pp. 43-46

331. Dž. Belkić, Quantum-Mechanical Signal Processing and Spectral Analysis (The Institute of Physics (Publishing, Bristol, 2005)

332. W.H. Press, B.P. Flannery, S.A. Teukolsky, W.T. Vetterling, Numerical Recipes in Fortran 77: The Art of Scientific Computing, 2nd edn. (Cambridge University Press, Cambridge, 1999)

333. C. Moler, Cleve's corner: Roots of polynomials, that is. MathWorks Newslett. 5(1), 8-9 (1991)

334. A. Edelman, H. Murakami, Polynomial roots from companion matrix eigenvalues. Math. Comput. 64, 763-776 (1995)

335. A. Girard, Invention Nouvelle en L'Algèbre (Guillaume Iansson Blaeuw, Amsterdam, 1629), English translation, in The Early Theory of Equations: On Their Nature and Constitution: Translations of Three Treatises by Viète, Girard, and De Beaune, ed. by R. Schmidt, E. Black (Golden Hind Press, Annapolis, Maryland, 1986) 